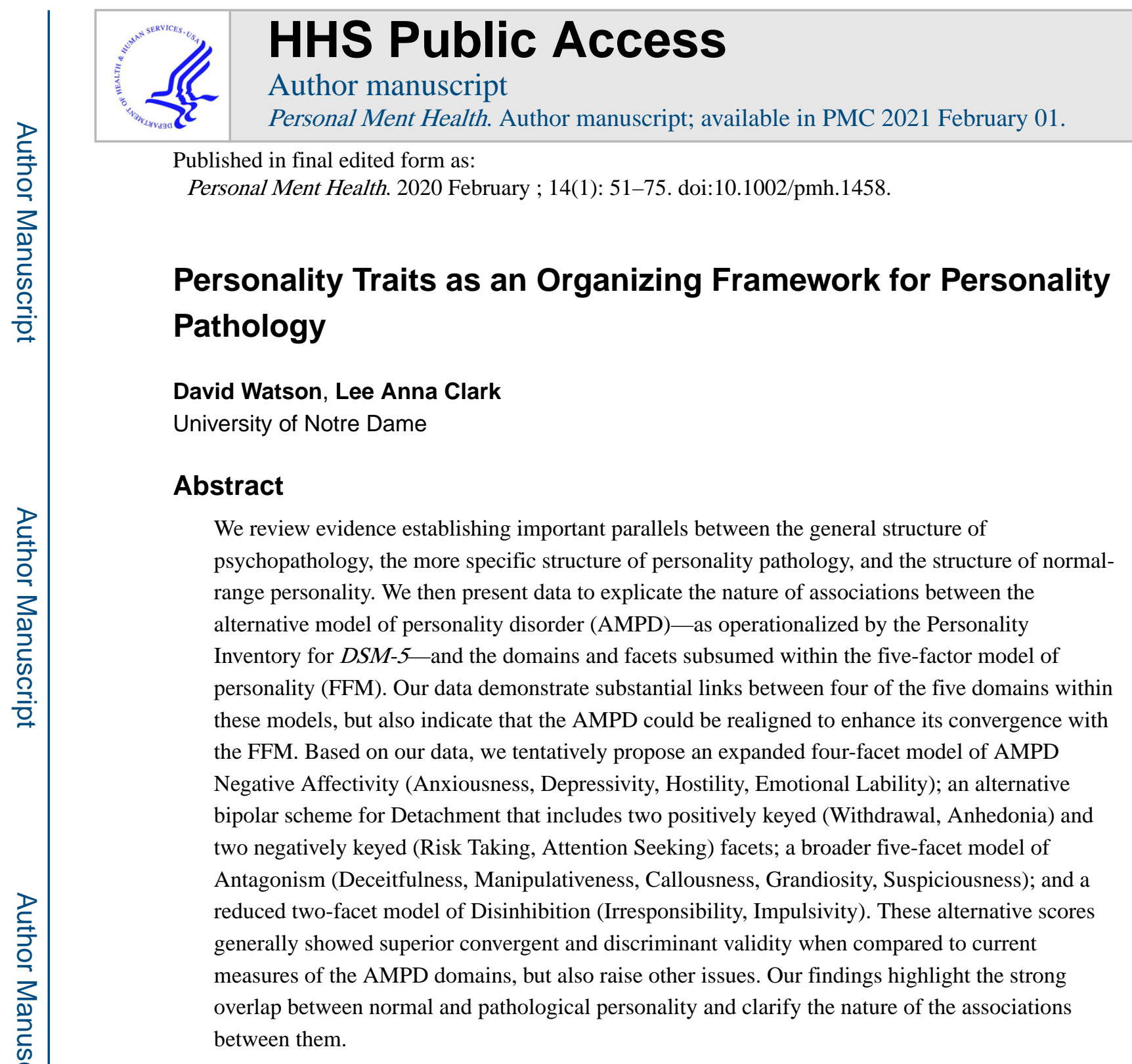

\title{
Keywords
}

alternative model of personality disorder; five-factor model of personality; negative affectivity; neuroticism; detachment; extraversion; antagonism; agreeableness; disinhibition; conscientiousness

There are important similarities between the higher order structures of personality and psychopathology. More specifically, it is increasingly recognized that there are clear parallels between the quantitative structure of psychopathology, the more specific structure of personality pathology, and the higher order structure of normal personality traits. ${ }^{1-3}$

The goal of this paper is to clarify the links among these related structures, thereby explicating the nature of the associations between personality and psychopathology. We begin by summarizing evidence establishing important similarities between the higher order

Correspondence concerning this paper should be addressed to David Watson, Department of Psychology, 390 Corbett Family Hall, University of Notre Dame, Notre Dame, IN, 46556, USA. db.watson@nd.edu. 
structures of psychopathology, personality pathology, and personality. We then examine more closely associations between broad domains of normal-range personality—as assessed primarily by the prominent five-factor model $\left(\mathrm{FFM}^{4-7}\right)$ —and personality pathology.

\section{Review of Relevant Evidence}

\section{General Structure of Psychopathology}

Identification of spectra.-A large number of studies have examined the quantitative structure of psychopathology and consistently have identified two overarching dimensions that model the comorbidity/covariation among many common mental disorders: Internalizing and Externalizing. ${ }^{1}$ The Internalizing dimension models the comorbidity among a broad range of conditions, including depressive, anxiety, trauma-related, obsessivecompulsive, and eating pathology. In contrast, Externalizing captures the covariation among conditions such as substance use problems, antisocial behavior, oppositional defiance, intermittent explosive behavior, and attention-deficits/hyperactivity. These higher order spectra have been identified in children, adolescents, and adults, and in a wide variety of cultures. ${ }^{8-11}$ In addition, there is evidence demonstrating the existence of two distinct subfactors within Externalizing, which Kotov et al. ${ }^{1}$ label Antagonistic Externalizing (e.g., narcissistic and paranoid personality pathology) and Disinhibited Externalizing (e.g., substance-related problems).

More recent data have identified an additional Thought Disorder spectrum. This dimension captures relations among symptoms of psychosis, mania and odd/eccentric personality pathology (e.g., schizotypy). This spectrum also has now been well replicated in both children and adults. ${ }^{1,8,12-14}$

Finally, there is more limited evidence to support two other higher order spectra. First, the Somatoform spectrum models relations between such conditions as somatic symptomatology and illness anxiety. ${ }^{1,13}$ Second, Detachment captures associations between such symptoms as interpersonal anxiety and social withdrawal, as well as related forms of personality pathology (e.g., avoidant, schizoid, and dependent). ${ }^{1,14-16}$

Based on their review of this evidence, Kotov et al. ${ }^{1}$ recently proposed an integrative Hierarchical Taxonomy Of Psychopathology (HiTOP). The HiTOP model incorporates these six higher order spectra-Internalizing, Antagonistic Externalizing, Disinhibited Externalizing, Thought Disorder, Detachment, and Somatoform—in a broader multilevel framework. We will not consider the Somatoform spectrum, which has received the least amount of support to date, further in this paper. The five remaining spectra are listed in the first column of Table 1.

Relations to personality.-Researchers quickly noted key similarities between these psychopathology spectra and the general structure of personality. Three distinct but interrelated models of personality are most relevant: First, the "Big Three" model consists of the higher order dimensions of Neuroticism, Extraversion, and Dyscontrol. ${ }^{4}$ Second, the highly influential FFM includes the broad domains of Neuroticism (vs. Emotional Stability), Extraversion, Agreeableness, Conscientiousness, and Openness/Open-mindedness. ${ }^{4-7}$ 
Notably, the FFM Neuroticism and Extraversion domains essentially are equivalent to the corresponding dimensions in the Big Three. The third Big-Three dimension, Dyscontrol, reflects a combination of low FFM Agreeableness and low Conscientiousness. FFM Openness is not directly modeled in the Big Three. Finally, the Personality Psychopathology Five (PSY-5 ${ }^{17}$ ) contains the broad dimensions of Negative Emotionality (similar to FFM Neuroticism), Introversion (low FFM Extraversion), Aggressiveness (low FFM Agreeableness), Disconstraint (low FFM Conscientiousness), and Psychoticism (which has a more complex association with the FFM). The FFM domains are listed in the final column of Table 1.

Evidence indicates that these basic personality dimensions play a key role in the emergence of the HiTOP spectra. That is, core Internalizing disorders all tend to be strongly related to Neuroticism, ${ }^{18}$ such that this spectrum broadly reflects individual differences on this trait. 3,19-21 Similarly, the overarching Externalizing spectrum is strongly linked to individual differences in the Big Three dimension of Dyscontrol; its Antagonistic and Disinhibited subfactors are more specifically associated with the FFM domains of low Agreeableness and low Conscientiousness, respectively. 3,19,21,22 Detachment reflects low levels of Extraversion. ${ }^{2-3}$ Finally, Thought Disorder is strongly related to PSY-5 Psychoticism. ${ }^{1,23}$ Its relation to FFM Openness is more complex; we revisit this issue in a later section.

\section{Structure of Personality Pathology}

DSM-5 AMPD.-Traditional categorical models of personality disorder suffer from several widely acknowledged problems, including low reliability, poor coverage, artificial diagnostic thresholds, substantial diagnostic comorbidity, and troublesome within-category heterogeneity. ${ }^{24-27}$ These widespread problems motivated the development of the alternative model for personality disorders (AMPD) in Section III of DSM-5. ${ }^{28}$ The AMPD incorporates a hierarchical trait structure that is strongly influenced by older models of personality, especially the FFM and PSY-5.

We consider the AMPD in greater detail later. For now, the key point is that it contains five higher order domains that strongly resemble those subsumed within (a) HiTOP and (b) standard trait models. ${ }^{1,3,23,29}$ These five AMPD domains are shown in the second column of Table 1; their labels clearly are borrowed from the personality literature and highlight their strong links to standard trait models. The AMPD domain of Negative Affectivity (vs. Emotional Stability) has been shown to be substantially associated with HiTOP Internalizing and FFM Neuroticism; Antagonism (vs. Agreeableness) is related to the HiTOP Antagonistic Externalizing spectrum and to low FFM Agreeableness; Disinhibition (vs. Conscientiousness) parallels HiTOP Disinhibited Externalizing and low FFM Conscientiousness; and Detachment (vs. Extraversion) is associated with HiTOP Detachment and low FFM Extraversion. Finally, Psychoticism (vs. Lucidity) is strongly linked to HiTOP Thought Disorder and PSY-5 Psychoticism; again, its association with FFM Openness is more complicated and is examined in detail later.

ICD-11 model.-The eleventh edition of the World Health Organization's International Classification of Diseases (ICD-1 $\left.11^{30}\right)$ introduced a trait-based model of personality 
pathology very similar to the AMPD. ${ }^{3,31-33}$ Like the AMPD, the $I C D-11$ model includes five higher order trait dimensions, which are presented in the third column of Table 1. Four of these domains - Negative Affectivity, Dissocial, Disinhibition, and Detachment—clearly correspond to those included in the AMPD. The ICD-11 model does not contain an equivalent of AMPD Psychoticism, as this content is included in the schizophrenia spectrum as Schizotypal Disorder, which actually serves to underscore the interrelation of personality and psychopathology. Instead, ICD-11 contains a dimension called Anankastia, which includes traits related to perfectionism, emotional and behavioral constraint, and obsessivecompulsive tendencies. This domain resembles the Compulsivity dimension that originally was part of the AMPD scheme. We have tentatively included it in Table 1 as the opposite end of the Disinhibition dimension, but data are accruing that suggest it may indeed be a distinct dimension 34,35 ; we return to this issue later.

The authors of this $I C D-11$ classification scheme explicitly acknowledge its strong similarities to both the AMPD and to the FFM. For example, Mulder et al. ${ }^{31}$ (note that this article was published before the $I C D-11$ was finalized, so some terminology differs slightly from the final version) state:

Interestingly, while the ICD-11 domains were derived independently, they are similar to four of the alternative DSM-5 domains. Negative Affective is similar to negative emotional, Detachment is similar to detachment, Dissocial is similar to antagonistic, and Disinhibition is similar to disinhibition. The major difference is that the proposed fifth domain is Anankastic in the ICD-11 system, while in DSM-5, this domain is labeled psychotic. The ICD-11 domains also map on to four of the five Big Five traits. Negative Affective with neuroticism, Detachment with low extraversion, Dissocial with low agreeableness, Disinhibited with low conscientiousness, and Anankastic with high conscientiousness. (p. 85) ${ }^{31}$

Thus, the ICD-11 scheme contains distinct domains containing maladaptive traits reflecting low and perhaps also high Conscientiousness - although, as mentioned earlier, Anankastia may instead be a distinct dimension, a point we return to in the General Discussion section.

\section{Evolution of the AMPD}

\section{Overview}

This brief review has highlighted substantial parallels between the higher order structures of psychopathology, personality pathology, and normal-range personality. These structural similarities are important and merit closer scrutiny. An intensive comparison of all of these models is beyond the scope of this paper. Consequently, we focus primarily on the relation between normal-range personality and personality pathology, as operationalized in the AMPD. To explicate this relation, we examine the Personality Inventory for DSM-5 (PID- $5^{23}$ ), a psychometric instrument that (a) was created specifically to assess the traits subsumed within the AMPD and (b) played a crucial role in the evolution of the AMPD. In this regard, one unusual aspect of the AMPD - at least in the context of standard psychiatric taxonomies, such as the $D S M$ - is that extensive psychometric data were collected to revise and refine it. 
The following sections focus on two related questions. First, how closely do the higher order domains of the AMPD actually parallel those of the FFM? Second, can we realign the facets subsumed within the AMPD domains so that they more closely parallel those of the FFM?

\section{Early Models}

Initial model.-Krueger et al. ${ }^{21,23}$ provide an extensive discussion of the creation of the PID-5 and its role in the evolution of the AMPD. The PID-5 was developed by members of the DSM-5Personality and Personality Disorders Workgroup and workgroup advisors. Item writing was based on an initial model consisting of 37 facets $^{23}$, which were organized into six higher order domains. This initial model included the five current AMPD domains, plus an additional higher order dimension of Compulsivity. As noted by Krueger et al. ${ }^{21}$, this sixth domain contained "some elements of excessive FFM conscientiousness" (p. 326) and, therefore, subsumed content similar to the Anankastia dimension in the ICD-11 model.

Preliminary model.-The PID-5 item pool was examined in three rounds of data collection. ${ }^{21.23}$ The resulting data led to two major changes in both the PID-5 and the AMPD. First, the number of facets was reduced from 37 to 25. Second, Compulsivity was dropped as a separate dimension; its component traits either were eliminated or moved to another domain.

These changes produced what we will call the Preliminary Model of the AMPD, which is presented in the first column of Table 2. This structure consisted of 25 facets that were grouped into five higher order domains, but the nature of these placements differ somewhat from subsequent schemes. As shown in Table 2, Negative Affectivity had seven component traits; Detachment, Antagonism, and Disinhibition each had five apiece; and Psychoticism contained three facets. Each facet was placed in only one domain; that is, there were no cross-listings in this model.

As noted, the Preliminary Model emerged empirically from structural evidence collected by the DSM-5 Personality and Personality Disorders Workgroup. This model clearly taps a wide range of content that overlaps substantially with standard personality structures, such as the FFM. Viewed solely from the perspective of these normal range models ${ }^{5-7}$, however, some of the facets appear to be misplaced. For example, most FFM models locate Depressivity within Neuroticism/Negative Affectivity, rather than within Detachment/ Extraversion. Moreover, Suspiciousness is more typically placed within Antagonism/ Agreeableness, rather than Detachment/Extraversion. More generally, these initial facet assignments do not align optimally with their corresponding placements in the FFM.

DSM-5 Section III Model-Structural analyses also revealed that several PID-5 facet scales cross-loaded on more than one higher order factor. ${ }^{23,36}$ This led to the revised model that is shown in the second column of Table 2. Because this is the structural scheme that actually is presented in Section III of DSM-5 (see Table 3, pp. 779-781), we have labeled it as such in Table 2. This revised scheme is the same as the Preliminary Model, except that four facets are cross-listed in two domains. Specifically, Depressivity and Suspiciousness now are included as facets of both Negative Affectivity and Detachment; Hostility is listed 
as a component of both Negative Affectivity and Antagonism; and Restricted Affectivity is a marker of both high Detachment and low Negative Affectivity.

However, the facets still do not appear to be optimally aligned in the Section III model relative to the FFM. For instance, Suspiciousness still is not placed within Antagonism. Moreover, although the decision to include cross-listings may have addressed some problems (e.g., Depressivity now is appropriately included as a facet of Negative Affectivity), it created others. Most notably, the existence of shared facets artifactually builds in some overlap between the domains.

\section{Triadic Model}

The situation is further complicated by the fact that the PID-5 materials provided on the American Psychiatric Association website recommend yet another set of guidelines for scoring these domains (see: https://www.psychiatry.org). This alternative scheme has been used in several studies ${ }^{37,38}$ and is listed in the third column of Table 2 . As can be seen, this reduced scheme involves scoring three core facets for each domain; hence, we refer to it as the Triadic Model.

The Triadic Model eliminates problems associated with cross-listings. At the same time, however, it significantly reduces the scope of most of the domains. For instance, we suggested earlier that Depressivity and Suspiciousness were misclassified in the Preliminary and DSM-5 Section-III models; here, they simply are ignored entirely.

Summary.-The AMPD clearly contains a wide range of content that overlaps substantially with the FFM. At the same time, however, none of the reviewed models appears to align the facets optimally vis-à-vis their corresponding placements in the FFM. Put differently, we can increase the convergence between the AMPD and the FFM structures by realigning the facets to maximize the similarity between them. In the following section, we examine relations between the AMPD and various personality instruments, and then use these data to suggest an alternative scoring scheme for most of the AMPD domains.

\section{Relations between the FFM and AMPD}

\section{Participants and Measures}

Participants.-We present data from three samples. Sample 1 consists of adults living in the greater South Bend metropolitan area $^{7}$; they were recruited for two online data sessions that were completed an average of 6.2 days apart. In the first session, 375 individuals completed both the PID-5 and the Faceted Inventory of the Five-Factor Model (FI-FFM ${ }^{7}$ ). In the second session, 335 participants were assessed with the Big Five Inventory $\left(\mathrm{BFI}^{39}\right)$.

Sample 2 was composed of 439 adults from the greater South Bend metropolitan area. ${ }^{7}$ Data were collected in two 3-hour sessions that were conducted at the Center for Advanced Measurement of Personality and Psychopathology (CAMPP). Session 1 included three comprehensive hierarchical trait instruments: the FI-FFM, the NEO Personality Inventory-3 (NEO-PI-3 ${ }^{5}$ ), and the Revised HEXACO Personality Inventory (HEXACO ${ }^{40}$ ). Session 2, held roughly 3 weeks later (mean interval $=20.3$ days), included an extensive battery of self- 
report psychopathology measures, including the PID-5; 411 participants completed this second session. We report data here on 411 (FI-FFM), 405 (NEO-PI-3), and 405 (HEXACO) participants.

Sample 3 (reported in Suzuki et al. ${ }^{41}$ ) consisted of 3,517 participants who completed both the PID-5 and the International Personality Item Pool NEO-PI R (IPIP-NEO ${ }^{42}$ ). Suzuki et al. ${ }^{41}$ present correlations between the IPIP-NEO facets and the PID-5 facet scales in a series of supplemental tables (see their Supplemental Tables B.1 through B.5). These results broaden the scope of our analyses by providing data based on another FFM instrument in a very large sample.

Measures.-Participants in all three samples were assessed on the PID-5. ${ }^{23}$ The PID-5 consists of sentences that are rated on a 4-point scale ranging from very false or often false to very true or often true. Respondents in the second and third samples completed the full, 220-item PID-5; however, three suicidality items were dropped in the version administered in the first sample, yielding a reduced 11-item version of the Depressivity scale.

Participants in Samples $1(N=375)$ and $2(N=411)$ completed the FI-FFM ${ }^{7}$, a factor analytically derived inventory that assesses specific lower order traits within the FFM framework. The items are sentences that are rated on a 5-point Likert scale ranging from strongly disagree to strongly agree. The FI-FFM contains 207 items and 22 facet scales (five apiece for neuroticism, extraversion, and conscientiousness; four for agreeableness; three for openness). Because respondents in these two samples were assessed on slightly different versions of the PID-5, we standardized the scale scores on a within-sample basis and then combined them to permit a single overall analysis. However, 27 of the Sample 2 participants also were included in Sample 1 and completed the PID-5 as part of that earlier study. Consequently, their PID-5 responses were dropped from Sample 2, yielding 384 nonoverlapping observations (overall $N=759$ for these analyses).

Personality assessment in Sample 2 also included the NEO-PI-3 and HEXACO. The NEO$\mathrm{PI}-3^{5}$ is an updated version of the widely used NEO PI-R. ${ }^{43}$ The only change was that 38 items were revised to lower the reading level, making the instrument more appropriate for younger examinees and adults with lower educational levels. The instrument consists of 240 items that are answered on a 5-point Likert scale ranging from strongly disagree to strongly agree. Each higher order FFM domain is assessed using six 8-item facet scales.

The HEXACO ${ }^{40}$ contains 100 sentences that are rated using a 5-point Likert scale ranging from strongly disagree to strongly agree. The instrument consists of 25 four-item scales that are organized into six higher order domains (Honesty-Humility, Emotionality, Extraversion, Agreeableness, Conscientiousness, and Openness). Each domain consists of four facets; the final scale (Altruism) is interstitial and is not scored on any domain. Previous joint factor analyses of the FI-FFM, NEO-PI-3, and HEXACO domain scales in Sample 2 established that the six higher order HEXACO domain scales can be mapped systematically within the FFM. ${ }^{44}$ Specifically, HEXACO Honesty-Humility and Agreeableness both are strong markers of Agreeableness; HEXACO Emotionality is a substantial indicator of Neuroticism; and the HEXACO Extraversion, Conscientiousness, and Openness scales help to anchor 
corresponding factors. Consequently, these scales will be used as additional indicators of the FFM domains in our analyses.

As noted, the Sample 3 participants were assessed using the IPIP-NEO. ${ }^{42}$ This instrument is modeled closely on the NEO PI-R and contains parallel 10-item scales designed to measure the same 30 lower order facets (six facets for each FFM domain). Further information regarding this measure can be obtained on the IPIP website (http://ipip.ori.org).

Finally, participants in Sample 1 completed the 44 -item $\mathrm{BFI}^{39}$, which contains 8-item scales assessing Neuroticism and Extraversion, a 10-item Openness scale, and 9-item measures of Agreeableness and Conscientiousness. The items are short phrases that are rated on a 5-point Likert scale ranging from strongly disagree to strongly agree.

\section{FFM Domain Analyses}

Relations with PID-5 domains.-How strong is the convergence between the structures defined by the FFM and AMPD? To answer this question, we first considered relations between the FFM domains and PID-5 domains (see Table 3); the latter were scored based on the Triadic Model (see Table 2; i.e., as the average of the three core facets for each domain).

Consistent with previous research ${ }^{3,29}$, these data indicate a generally strong level of convergence between four of the five domains. PID-5 Negative Affectivity was strongly positively related to all three Neuroticism scales ( $I$ s ranged from .70 to .77 ); it was more moderately related to HEXACO Emotionality $(r=.44)$ and, in fact, was almost as strongly linked to HEXACO Extraversion $(r=-.42)$, indicating that Emotionality subsumes content that is somewhat less relevant to this domain of the AMPD. PID-5 Detachment was negatively correlated with all four indicators of Extraversion ( $I$ s ranged from -.53 to -.67), but also correlated strongly with the Neuroticism scales of the FI-FFM and NEO-PI-3. PID-5 Disinhibition was strongly and consistently negatively related to Conscientiousness (Is ranged from -.58 to -.70 ), but also had strong correlations with FI-FFM and NEO-PI-3 Neuroticism. PID-5 Antagonism had strong negative associations with the Agreeableness scores from both the FI-FFM $(r=-.64)$ and NEO-PI-3 $(r=-.59)$. Replicating the findings of Ashton et al. ${ }^{45}$, it had a much stronger negative link to HEXACO Honesty-Humility ( $r=$ $-.55)$ than to HEXACO Agreeableness $(r=-.39)$. Finally, it is noteworthy that PID-5 Antagonism correlated only moderately with BFI Agreeableness $(r=-.48)$, suggesting that this scale lacks some of the AMPD-relevant content that is assessed by other indicators of this domain.

As noted, several discriminant correlations are quite high, suggesting some potentially serious problems in linking the two structures at the higher order level. In particular, PID-5 Detachment and Disinhibition were almost as strongly correlated with FI-FFM and NEOPI-3 Neuroticism as with their expected corresponding domain scales (Extraversion and Conscientiousness, respectively). We revisit these problems later.

Finally, PID-5 Psychoticism did not display consistently strong associations with any FFM domain. Overall, its most substantial relations were with FI-FFM Neuroticism $(r=.53)$ and NEO-PI-3 Neuroticism $(r=.49)$. It had moderate negative links to indicators of both 
Agreeableness (Is ranged from - .35 to -.46 ) and Conscientiousness ( $I$ s ranged from -.30 to -.40), but was weakly related to both Extraversion ( 1 s ranged from -.02 to -.26) and Openness (Is ranged from .01 to .17). Conversely, unlike the other FFM domains, indicators of Openness consistently displayed relatively weak relations in these data; in fact, the strongest association was only -.20 (between BFI Openness and Detachment, and between HEXACO Openness and Negative Affectivity). However, these domain-based correlations may mask stronger associations at the lower order level, which we examine subsequently.

Relations with PID-5 facets.-Although the data reported in Table 3 are promising, they also suggest some non-trivial problems in linking the two structures. As a first step in identifying potential sources of these problems, we examined relations between the FFM domains and the individual PID-5 facet scales in Sample 2. We began by conducting a principal factor analysis (using squared multiple correlations as the initial communality estimates) of the NEO-PI-3 and FI-FFM domain scales; we extracted five factors and rotated them to oblique simple structure using promax (power $=3$ ). The five resulting factors (see Table 4) clearly can be interpreted as Conscientiousness, Agreeableness, Extraversion, Neuroticism, and Openness, respectively. We computed regression-based factor scores to model these five dimensions. Correlations among these factor scores ranged from .55 (between Extraversion and Openness) to -.71 (between Conscientiousness and Neuroticism).

Table 5 presents correlations between these factor scores and the individual PID-5 facet scales in Sample 2; the PID-5 facets are arranged based on their status in the Triadic Model (e.g., the three facets of Negative Affectivity are grouped together). Five aspects of these data are noteworthy. First, the convergent correlations varied widely in magnitude, demonstrating that some facets have substantially stronger links to the FFM than others. In particular, the PID-5 Detachment facet correlations ranged from -.28 to -.59 with Extraversion, and the Antagonism facet correlations ranged from - .39 to - .64 with Agreeableness.

Second, the Detachment facets failed to show a clear convergent/discriminant pattern in these data; in fact, all three facets correlated as strongly with Neuroticism ( $I$ s ranged from .35 to .66 ; mean $r=.52$ ) as they did with Extraversion ( $r$ s ranged from -.28 to -.59; mean $r=-.47)$. Intimacy Avoidance is particularly problematic, as it was weakly related to Extraversion $(r=-.28)$, and had correlations similar in magnitude with Neuroticism $(r$ $=.35)$, Agreeableness $(r=-.26)$, and Conscientiousness $(r=-.28)$.

Third, the Disinhibition facet of Distractibility appears to be particularly responsible for the strong association with Neuroticism that was observed in Table 3. In fact, it correlated as strongly with FFM Neuroticism $(r=.64)$ as it did with Conscientiousness $(r=-.63)$. That said, both Irresponsibility and Impulsivity also correlated almost as strongly with Neuroticism ( $r=.53$ and .48 , respectively) as with Conscientiousness ( $r=-.56$ and -.50 , respectively), albeit at a somewhat weaker level.

Fourth, none of the Psychoticism facets showed a clear convergent/discriminant pattern. They all were moderately to strongly associated with Neuroticism ( $I$ s ranged from .37 
to .53 ), moderately negatively related to Agreeableness ( $I \mathrm{~s}$ ranged from -.35 to -.44 ) and Conscientiousness ( $I$ s ranged from -.27 to -.37 ), and weakly related to both Extraversion ( $I \mathrm{~s}$ ranged from -.06 to -.18 ) and Openness (Is ranged from -.00 to .10 ).

Finally, some scales that currently are not scored as facets in the Triadic Model actually correlated quite strongly with certain FFM domains. Most notably, PID-5 Depressivity correlated .68 with Neuroticism, whereas Callousness was strongly negatively related ( $r=$ -.66) to Agreeableness.

\section{FFM Facet Analyses}

Overview.-Next, we examine relations between the PID-5 and the individual facet scales subsumed within each FFM domain. We used two criteria in selecting PID-5 facets for inclusion in these analyses. First, to clarify how the current operationalization of the AMPD relates to the FFM, we included the three component facets that define the corresponding domain in the Triadic Model (see Table 2); we also included the Triadic Model domain score for comparison purposes. Second, we selected PID-5 scales that had particularly strong and specific associations with individual FFM traits in that domain.

Neuroticism.-Table 6 presents correlations between FFM Neuroticism facets and selected PID-5 scales. It is noteworthy that three PID-5 scales show a clear convergent/ discriminant pattern vis-à-vis similarly named facet scales from the FI-FFM, NEO-PI-3, and IPIP-NEO. Specifically, PID-5 Anxiousness correlated most strongly with indicators of trait anxiety (Is ranged from .68 to .79), PID-5 Depressivity was highly related to facet scales assessing depression ( $I$ s ranged from .69 to .80), and PID-5 Hostility was strongly associated with measures of anger and hostility ( 1 s ranged from .65 to .76). Thus, all four instruments contain highly correlated scales assessing the same three lower order traits. PID-5 Emotional Lability - which is classified as a facet of Negative Affectivity in every version of the AMPD (see Table 2) - was strongly but nonspecifically related to these same facet scales, with coefficients ranging from .53 to .65 across inventories. It also was substantially associated with both FI-FFM Somatic Complaints $(r=.52)$ and IPIP-NEO Vulnerability $(r$ $=.62$ ). In this respect, it may be considered the best single indicator of this domain. In contrast, Separation Insecurity - which also is classified as a facet of Negative Affectivity in every instantiation of the AMPD—was more moderately linked to Neuroticism. Its strongest association was with FI-FFM Depression $(r=.51)$. Finally, the HEXACO showed only one association $2.50-$ namely, the .52 correlation between Anxiety and PID-5 Anxiousness.

Based on these findings, we suggest an alternative model for the AMPD Negative Affectivity domain consisting of four PID-5 facets: Anxiousness, Depressivity, Hostility, and Emotional Lability. We examine the correlates of this revised domain score in a later section.

Extraversion.-Table 7 presents parallel data for Extraversion. Intimacy Avoidance consistently exhibited relatively weak associations with the lower order traits within this domain. In fact, it had only four correlations with an absolute value $\geq .30$, and none as high as .40. These results suggest that to increase convergence with FFM Extraversion, this scale should be dropped as an indicator of AMPD Detachment. 
In contrast, the other two Detachment facets consistently displayed strong links to specific components of Extraversion. PID-5 Withdrawal was strongly negatively related to indicators of sociability, friendliness and gregariousness (e.g., $r=-.79$ with IPIP-NEO Friendliness, -.68 with FI-FFM Sociability, -.56 with HEXACO Sociability, and -.54 with NEO-PI-3 Gregariousness), whereas Anhedonia was substantially linked to low positive emotionality (e.g., $r=-.69$ with HEXACO Liveliness, -.67 with FI-FFM Positive Temperament, -.62 with IPIP-NEO Cheerfulness, -.62 with NEO-PI-3 Positive Emotions).

Importantly, the Table 7 data also illustrate a significant limitation of the current AMPD operationalization of Detachment. Unlike other FFM domains, Extraversion shows both negative and positive associations with psychopathology. 46,47 The current AMPD operationalization focuses entirely on the former and completely ignores the latter. Table 7 further establishes that two PID-5 scales—Risk Taking and Attention Seeking —have substantial positive associations with specific components within this domain. For instance, Risk Taking correlated .73 with IPIP-NEO Excitement-Seeking, .56 with FI-FFM Venturesomeness, and .56 with NEO-PI-3 Excitement-Seeking. Attention Seeking had its strongest associations with FI-FFM Ascendance $(r=.56)$, IPIP-NEO Excitement-Seeking $(r$ $=.52$ ), IPIP-NEO Assertiveness $(r=.49)$, and FI-FFM Venturesomeness $(r=.45)$.

Based on these results, we suggest a fundamentally different approach to modeling AMPD Detachment (which perhaps would be more accurately labeled as Pathological Introversion in its current form). Our proposed alternative includes two positively keyed facets (Withdrawal and Anhedonia) as well as two negatively keyed traits (Risk Taking and Attention Seeking). As Table 7 shows, this alternative model is more effective in capturing the full range of content subsumed within the Extraversion domain.

Agreeableness-Corresponding results for Agreeableness are presented in Table 8. It is important to note that the three current facets of Antagonism (see Table 2) all were substantially related to traits within this domain. Most notably, Deceitfulness and Manipulativeness both were strongly negatively linked to FFM indicators of straightforwardness and morality (viz., FI-FFM Straightforwardness, NEO-PI-3 Straightforwardness, IPIP-NEO Morality), with correlations ranging from -.60 to -.78. They correlated more moderately with HEXACO Sincerity and Fairness (Is ranged from -.33 to -.54$)$. Grandiosity was moderately associated with most aspects of Agreeableness, but exhibited strong negative relations with IPIP-NEO Modesty $(r=-.62)$ and Morality $(r=$ $-.55)$.

At the same time, however, the Table 8 data establish that these three facets fail to capture all of the relevant content subsumed within this trait domain, such that we can increase FFMrelated coverage by specifying additional markers of Antagonism. Two PID-5 scales seem particularly promising. First, like Deceitfulness and Manipulativeness, Callousness also had strong negative associations with scales assessing straightforwardness and morality $(r=-.62$ with IPIP-NEO Morality, -.62 with FI-FFM Straightforwardness, -.55 with NEO-PI-3 Straightforwardness). In addition, it was strongly negatively related to scales assessing empathy and altruism (e.g., $r=-.65$ with IPIP-NEO Altruism, -.59 with IPIP-NEO Sympathy, -.53 with FI-FFM Empathy). Second, the PID-5 Suspiciousness scale showed a 
strong, specific negative association with the Trust scales from the IPIP-NEO $(r=-.66)$, FIFFM $(r=-.65)$, and NEO-PI-3 $(r=-.62)$.

Two other PID-5 scales displayed strong links to at least some aspects of Agreeableness. Hostility had particularly strong negative associations with IPIP-NEO Cooperation ( $r=$ -.66), HEXACO Patience ( $r=-.63$ ), and NEO-PI-3 Compliance $(r=-.58)$. Attention Seeking correlated -.58 with IPIP-NEO Modesty, -.57 with IPIP-NEO Morality, and -.51 with FI-FFM Modesty. However, we already have assigned both of these PID-5 scales to another AMPD domain (Hostility to Negative Affectivity, Attention Seeking as a negativelykeyed facet of Detachment), so they will not be considered further here. Consequently, we propose a broadened model of Antagonism that consists of five facets: Deceitfulness, Manipulativeness, Callousness, Grandiosity, and Suspiciousness.

Conscientiousness.-Table 9 presents associations with Conscientiousness. All three facets of Disinhibition displayed strong inverse associations within this domain. Moreover, they related to different aspects of the domain. PID-5 Irresponsibility was strongly negatively related to the Dutifulness scales of the IPIP-NEO $(r=-.71)$, FI-FFM $(r=-.66)$, and NEO-PI-3 $(r=-.53)$. In contrast, Impulsivity was inversely related to indicators of cautiousness and prudence ( $r=-.76$ with IPIP-NEO Cautiousness, -.70 with FI-FFM Deliberation, - .65 with NEO-PI-3 Deliberation, -.61 with HEXACO Prudence). Finally, Distractibility was most strongly linked to the Self-Discipline scales of the IPIP-NEO ( $r=$ $-.68)$, FI-FFM $(r=-.65)$, and NEO-PI-3 $(r=-.62)$.

These results suggest that the current conceptualization of AMPD Disinhibition in the Triadic Model is reasonable. One complication, however, is the strong correlation between Distractibility and Negative Affectivity $(r=.65)$ that was observed in Table 5. We consider the possibility of dropping Distractibility as a facet of Disinhibition subsequently.

Openness.-Table 10 reports associations between AMPD Psychoticism and FFM Openness. In interpreting these data, it should be noted that previous research has shown that these relations display considerable specificity at the facet level. ${ }^{41,48,49}$ For instance, Chmielewski et al. ${ }^{48}$ found that the NEO PI-R Fantasy scale was positively related to PID-5 Psychoticism, whereas the other Openness facets either had no relation, or were negatively related to this AMPD domain.

The Table 10 data further demonstrate the specificity of these relations. Most of the correlations are quite low. However, IPIP-NEO Imagination had moderate positive links to all three facets of Psychoticism ( $r=.40$ with Eccentricity, .34 with Perceptual Dysregulation, .33 with Unusual Beliefs \& Experiences). In addition, FI-FFM Nontraditionalism and NEO-PI-3 Fantasy both correlated moderately with Eccentricity ( $r$ $=.28$ and .21 , respectively). Replicating previous findings, Table 10 also contains some significant negative associations. For instance, NEO-PI-3 Actions correlated -.17 with Perceptual Dysregulation, NEO-PI-3 Values correlated -.15 with Unusual Beliefs and Experiences, and IPIP-NEO Artistic Interests correlated -.11 with Perceptual Dysregulation. 
These results establish some level of overlap between AMPD Psychoticism and FFM Openness. Nevertheless, the situation here is quite different from that seen in the other four domains. None of the Table 10 correlations represents a large effect size ${ }^{50}$; indeed, the strongest association is only .40. Furthermore, as shown in Table 5, all three Psychoticism facets actually correlated more strongly with domains other than Openness. The nonspecific nature of these relations is further illustrated by the IPIP-NEO results reported in the supplemental tables of Suzuki et al. ${ }^{41}$. In their data, for example, PID-5 Perceptual Dysregulation correlated -.54 with Dutifulness (a facet of Conscientiousness), .49 with Depression (a facet of Neuroticism), and -.49 with Morality (a facet of Agreeableness); Unusual Beliefs and Experiences correlated -.38 with Morality and -.35 with Dutifulness; and Eccentricity correlated -.53 with Dutifulness and -.51 with Morality.

Consequently, these data provide no real basis for rethinking the nature of Psychoticism. We therefore suggest no changes to the scoring of this domain.

\section{Analyses of the Revised AMPD Domains}

Negative Affectivity, Detachment, and Antagonism.-We have proposed revised scoring schemes for PID-5 Negative Affectivity, Detachment, and Antagonism. How do these rescored domains compare to the current versions contained in the Triadic Model? To answer this question, Table 11 presents correlations between (a) the current and revised versions of these AMPD domains and (b) the higher order FFM scales. As in the Triadic Model, the revised PID-5 scores were calculated by averaging the proposed facets-four apiece for Negative Affectivity and Detachment, five for Antagonism—for each domain.

The revised Negative Affectivity domain score consistently shows slightly better convergence with Neuroticism. Correlations with Neuroticism increased from .77 to .83 in the FI-FFM; from .70 to .74 in the NEO-PI-3; and from .76 to .78 in the BFI. However, the correlation with HEXACO Emotionality decreased somewhat from .44 to .30 ; moreover, the correlation with Extraversion increased from -.42 to -.53. Overall, our expanded version of the domain appears to be a slight improvement over that currently included in the Triadic Model.

Our revised version of Detachment represents a more dramatic improvement, at least in the analyses involving the FI-FFM and the NEO-PI-3. Here, the convergent correlations have increased substantially, from -.53 to -.76 in the FI-FFM, and from -.60 to - .74 in the NEOPI-3. Equally importantly, the troublesome discriminant correlations with Neuroticism are greatly improved: They have dropped from .57 to .29 in the FI-FFM, and from .57 to .33 in the NEO-PI-3. Thus, the revised PID-5 Detachment score now shows a strong and specific association with (low) Extraversion in both of these instruments. In contrast, the effects are much less dramatic in the BFI and HEXACO; here, the convergent correlations actually drop slightly from -.67 to -.61 (BFI), and from -.64 to -.62 (HEXACO). At the same time, however, the moderate discriminant correlations with Agreeableness essentially disappeared, dropping from -.43 to -.06 in the BFI, and from -.32 to -.03 in the HEXACO.

These discrepant results are easily explained. Our reconceptualization of Detachment emphasizes content—such as excitement seeking — that is much more prominent in the FI- 
FFM and NEO-PI-3 than it is in these other personality inventories. Consequently, it makes perfect sense that our revised scheme raises convergence substantially vis-à-vis the FI-FFM and NEO-PI-3, but not in relation to the BFI and HEXACO. These findings highlight the important point that results inevitably will vary somewhat across different conceptualizations of the FFM.

Finally, our expanded model of Antagonism consistently outperforms the current version in the Triadic Model in that the convergent correlations increase systematically: from -.64 to -.73 in the FI-FFM; from -.59 to -.68 in the NEO-PI-3; from -.48 to -.61 in the BFI; and from -.55 to -.57 (Honesty-Humility) and -.39 to -.49 (Agreeableness) in the HEXACO. However, it also must be acknowledged that the discriminant correlation with Neuroticism increased from .31 to .44 in the FI-FFM, and from .28 to .40 in the NEO-PI-3.

Disinhibition.-Next, we revisit the problem of Distractibility, a scale that correlates strongly with both Conscientiousness and Neuroticism (see Tables 5 and 9). As would be expected, dropping this scale as a facet of Disinhibition consistently improves the discriminant validity of the domain score. The discriminant correlation with Neuroticism drops from .62 to .52 (FI-FFM), from .64 to .54 (NEO-PI-3), and from .47 to .36 (BFI). Not surprisingly, however, the convergent correlations with Conscientiousness also decline: from -.70 to -.63 (FI-FFM), from - .65 to -.59 (NEO-PI-3), from - .70 to -.65 (BFI), and from -.58 to -.53 (HEXACO). Overall, the reduced two-scale version of Disinhibitionconsisting simply of Irresponsibility and Impulsivity—shows a somewhat clearer convergent/discriminant pattern than the current scheme contained in the Triadic Model. Which model is judged to be better ultimately depends on the relative weight that is assigned to convergent versus discriminant validity.

\section{Joint Factor Analyses}

As an additional test of the validity of our revised PID-5 scoring scheme, we conducted a combined structural analysis of the four parallel domains subsumed within both the FFM and AMPD. Specifically, we examined the structure jointly defined by (a) the FI-FFM Neuroticism, Extraversion, Agreeableness, and Conscientiousness facet scales and (b) the PID-5 scales included in our proposed scoring schemes for AMPD Negative Affectivity, Detachment, Antagonism, and Disinhibition. We subjected these scales to a principal factor analysis (using squared multiple correlations as the initial communality estimates). We extracted four factors and rotated them to oblique simple structure using promax (power = 3). This yielded a clear and well-defined solution; Table 12 presents loadings on these four factors. As can be seen, these factors clearly represent Neuroticism/Negative Affectivity, Antagonism vs. Agreeableness, Extraversion vs. Detachment, and Conscientiousness vs. Disinhibition, respectively. Thus, these FI-FFM and PID-5 scales jointly define a common Big Four structure.

All of the FI-FFM facet scales load strongly on their target factor, thereby replicating the internal structure of the instrument. ${ }^{7}$ For our purposes, however, the more important issue concerns the placement of the PID-5 scales within this joint structure. In this regard, it is important to note that all of the PID-5 scales loaded substantially (i.e., had a loading with an 
absolute value of .35 or greater) on their target factor. The PID-5 Negative Affectivity and Antagonism scales tended to be particularly strong markers of their respective factors (loadings range from .41 to .94 , mean $=.70$ ). Consequently, these results provide substantial support for our alternative scoring scheme.

At the same time, however, eight of the 16 PID-5 scales (50\%) had salient loadings on more than one factor, which suggests that they perhaps are best viewed as interstitial traits within the framework of the FFM. For example, PID-5 Hostility and Suspiciousness both split between Factors I and II; Attention Seeking, Risk Taking, and Withdrawal all split between Factors II and III; Anhedonia split between Factors I and III; Distractibility split between Factors I and IV; and Irresponsibility split between Factors II and IV. These more complex placements merit further attention in future research.

\section{General Discussion}

\section{Implications of the Findings}

We reviewed evidence establishing substantial parallels between the higher order structures of psychopathology, personality pathology, and normal-range personality-structural parallels that are important and warrant further investigation. Accordingly, we presented a series of analyses to explicate the nature of associations between the AMPD—as operationalized by the PID-5-and the FFM. Our results clearly demonstrate substantial convergence between four domains subsumed within these two structures: Negative Affectivity and Neuroticism, Detachment and (low) Extraversion, Antagonism and (low) Agreeableness, and Disinhibition and (low) Conscientiousness.

At the same time, however, our findings revealed that these associations are more complex than is typically acknowledged. We believe that these structural complexities merit greater attention and that understanding them is important for three reasons. First, they help to clarify the nature of relations between normal and pathological personality. For instance, our data indicate that Intimacy Avoidance, a facet of Detachment in the AMPD, actually has relatively weak and nonspecific associations with FFM domains and facets (see Tables 5 and 7). Moreover, our findings demonstrate that the current conceptualization of Detachment focuses almost entirely on characteristics that are negatively related to Extraversion, such as social aloofness (PID-5 Withdrawal) and low positive emotionality (PID-5 Anhedonia). As our data show, however, some aspects of Extraversion have important positive links to personality pathology, exhibiting moderate to strong associations with trait characteristics such as recklessness (PID-5 Risk Taking) and exhibitionism (PID-5 Attention Seeking); moreover, when the PID-5 and FI-FFM are co-factored, a bipolar factor emerges with these scales assessing opposite ends of the same dimension (see Table 12).

Second, our findings establish that many PID-5 scales assess the same lower order traits that are included in standard hierarchical measures of the FFM. For example, PID-5 Depressivity converges strongly with Neuroticism facet scales assessing dysphoria and low self-esteem (e.g., FI-FFM, NEO-PI-3, and IPIP-NEO Depression); PID-5 Withdrawal taps the opposite end of a dimension within Extraversion that is anchored by measures of gregariousness and sociability at the other pole (e.g., IPIP-NEO Friendliness, FI-FFM Sociability, NEO-PI-3 
Gregariousness, HEXACO Sociability); PID-5 Suspiciousness defines one pole of a bipolar continuum marked at the other end by indicators of trustfulness (e.g., FI-FFM, IPIP-NEO, and NEO-PI-3 Trust); and PID-5 Impulsivity is strongly negatively related to Conscientiousness scales tapping cautiousness (e.g., IPIP-NEO Cautiousness, FI-FFM Deliberation, NEO-PI-3 Deliberation, HEXACO Prudence).

Third, explicating these associations can point toward underlying mechanisms that may (a) cause certain forms of psychopathology and also (b) account for the comorbidity among them. For instance, Extraversion reflects individual differences in the behavioral activation system (BAS) and, consequently, in reward-seeking behavior. ${ }^{51-53}$ Several lines of research indicate that mania reflects heightened sensitivity of the BAS, such that vulnerable individuals are overly sensitive to minor signals of reward. ${ }^{54}$ This, in turn, helps to explain the positive associations between Extraversion and mania that have been reported in the literature. ${ }^{47,55}$ In a related vein, Johnson and colleagues ${ }^{56}$ link mania and various forms of externalizing psychopathology—including both narcissism and psychopathy-to individual differences in the dominance behavioral system (DBS), which they define as a biologically based system guiding "dominance motivation, dominant and subordinate behavior, and responsivity to perceptions of power and subordination" (p. 692) ${ }^{56}$ They summarize extensive data establishing that elevated levels of the DBS are associated with both increased reward sensitivity and traits related to extraversion (especially assertiveness/dominance).

\section{Issues for Future Research}

Alternative models of the FFM.-Future work in this area needs to address several important issues. First, as noted earlier, the nature of relations between personality and the AMPD varies somewhat across alternative conceptualizations of the FFM due to differences in assessed content. As shown in Table 3, for example, Antagonism had a stronger negative relation to FI-FFM Agreeableness $(r=-.64)$ and NEO-PI-3 Agreeableness $(r=-.59)$ than to BFI Agreeableness $(r=-.48)$. This difference likely is due to the fact that the BFI Agreeableness scale does not include the full range of content related to insincerity and amorality that is contained in these other instruments (as captured in scales such as FI-FFM and NEO-PI-3 Straightforwardness). Similarly, our proposed alternative version of Detachment correlated more strongly with Extraversion domain scores from the FI-FFM ( $r=$ $-.76)$ and NEO-PI-3 $(r=-.74)$ than from the HEXACO $(r=-.62)$ and the BFI $(r=-.61)$. This reflects the fact that our revised Detachment score contains content related to recklessness and excitement seeking that is modeled in the FI-FFM and NEO-PI-3, but not in these other inventories. ${ }^{7}$

This brings us to a more fundamental problem, namely, that we currently lack a consensual model of personality at the lower order level. ${ }^{47,57}$ This means that we can describe differences in content across measures, but are unable to evaluate them in any compelling way. For example, as already noted, the NEO-PI-3 and FI-FFM subsume content related to excitement seeking within Extraversion, whereas other instruments do not. Which model of Extraversion is better? Currently, we cannot provide a definitive answer to this question. Research on the links between adaptive and maladaptive personality will be hampered until we have achieved greater clarity regarding the lower order level of the FFM hierarchy. 
Alternative models of personality pathology.-Similar considerations apply to the conceptualization and assessment of personality pathology. Alternative models of personality pathology exist, and they inevitably will lead to somewhat different findings and conclusions. As shown in Table 1, for example, although the ICD-11 model for personality disorder is similar in many ways to the AMPD of DSM-5, there also are non-trivial differences between these two classification schemes. Perhaps the most important difference is the inclusion of anankastia in the $I C D-11$ model, a trait whose core feature is a narrow focus on a rigid standard of perfection and on behavioral and emotional control. As such, anankastia is conceptualized as distinct from disinhibition, not simply its opposite (contrary to where we tentatively placed it in Table 1). Although some aspects of each construct are, no doubt, negatively correlated, evidence is accruing both to support this view ${ }^{34,35}$ and to suggest that its opposite end may instead be openness to experience. ${ }^{58}$

Moreover, the results reported here are based on a single psychometric instrument, the PID-5. Other self-report measures of personality pathology are available and can be expected to yield different results. For instance, Simms and colleagues have developed another quasi-comprehensive measure of pathological traits, the Comprehensive Assessment of Traits relevant to Personality Disorder (CAT-PD ${ }^{59,60}$ ). Although the CAT-PD contains substantial content that overlaps with the PID-5, it also includes several scales (e.g., Health Anxiety, Domineering, Rudeness, Workaholism) containing content that the PID-5 does not directly capture.

More fundamentally, every model reflects its own biases and assumptions. The development of the PID-5-and the AMPD more generally — was guided by the belief that the FFM provided a valuable organizing framework for understanding personality pathology. ${ }^{21,23} \mathrm{We}$ believe that subsequent evidence, including the data we have reported here, has demonstrated the wisdom of this belief. Nevertheless, the fact remains that other sets of assumptions would have provided different starting points and ultimately yielded somewhat different models and measures.

The issue of interstitial traits.-The Table 12 results demonstrate that many of the PID-5 scales are interstitial, that is, have significant links to more than one FFM domain. This is one manifestation of a more general issue. Hopwood and Donnellan ${ }^{61}$ review evidence indicating that personality traits do not conform neatly to the factor analytic ideal of simple structure. Because of this, many personality scales are interstitial and do not fall cleanly within a single higher order domain. This is true both of the FFM and the AMPD. For example, our data clearly show that PID-5 Distractibility is interstitial, with strong links to both Disinhibition and Neuroticism/Negative Affectivity. Similarly, many FFM scales have significant links to more than one domain. For instance, measures of anger/hostility (e.g., NEO-PI-3 Angry Hostility, FI-FFM Anger Proneness) and trust (e.g., NEO-PI-3 and FI-FFM Trust) tend to be indicators of both elevated Neuroticism and low Agreeableness.

These interstitial traits create challenges when we attempt to link domains across models and measures. In our analyses, for example, we were confronted with the issue of whether or not to include Distractibility as a facet of Disinhibition: Including it enhances its convergent validity with Conscientiousness, but also reduces its discriminant validity vis-à-vis 
Neuroticism. Future research will need to confront these issues more fully to determine the best way to handle interstitial traits.

\section{Conclusion}

The accumulating data establish important connections between the higher order domains of psychopathology, personality pathology, and normal-range personality. Moreover, the data we have presented here highlight particularly strong associations between the FFM of normal personality and the AMPD of DSM-5. These substantial links are important and merit closer scrutiny in the future. We encourage other researchers to build on our findings to articulate more fully the joint structure of personality and psychopathology.

\section{Acknowledgments}

We thank Patrick Cruitt, Stephanie Ellickson-Larew, Mark Godding, Haley Heibel, Brittany Katz, Ana Kent, Katie Kraemer, Mallory Meter, Eunyoe Ro, John Souter, Kasey Stanton, Sara Stasik-O’Brien, Nadia Suzuki, and Elizabeth Yahiro for their help in the preparation of this manuscript. This research was supported by National Institute of Mental Health Grant R01-MH083830 to Lee Anna Clark.

\section{References}

1. Kotov R, Krueger RF, Watson D, Achenbach TM, Althoff RR, Bagby RM et al. The Hierarchical Taxonomy of Psychopathology (HiTOP): A dimensional alternative to traditional nosologies. $\mathbf{J}$ Abnorm Psychol; 2017 126: 454-477. [PubMed: 28333488]

2. Watson D, Ellickson-Larew S, Stanton K, Levin-Aspenson H. Personality provides a general structural framework for psychopathology: Commentary on "Translational applications of personality science for the conceptualization and treatment of psychopathology." Clin Psychol Sci Pract; 2016 23: 309-313.

3. Widiger TA, Sellbom M, Chmielewski M, Clark LA, DeYoung CG, Kotov R et al. Personality in a hierarchical model of psychopathology. Clin Psychol Sci; 2019 7: 77-92.

4. Markon KE, Krueger RF, Watson D. Delineating the structure of normal and abnormal personality: An integrative hierarchical approach. J Pers Soc Psychol; 2005 88: 139-157. [PubMed: 15631580]

5. McCrae RR, Costa PT, Martin TA. The NEO-PI-3: A more readable Revised NEO Personality Inventory. J Pers Assess; 2005 84: 261-270. [PubMed: 15907162]

6. Soto CJ \& John OP. The next Big Five Inventory (BFI-2): Developing and assessing a hierarchical model with 15 facets to enhance bandwidth, fidelity, and predictive power. J Pers Soc Psychol; 2017 113: 117-143. [PubMed: 27055049]

7. Watson D, Nus E, Wu KD. Development and validation of the Faceted Inventory of the Five-Factor Model (FI-FFM). Assess; 2019 26: 17-44.

8. Achenbach TM, Ivanova MY Rescorla LA. Empirically based assessment and taxonomy of psychopathology for ages 11/2-90+ years: Developmental, multi-informant, and multicultural findings. Compr Psychiatry; 2017 79: 4-18. [PubMed: 28356192]

9. Carragher N, Krueger RF, Eaton NR, Markon KE, Keyes KM, Blanco C et al. ADHD and the externalizing spectrum: direct comparison of categorical, continuous, and hybrid models of liability in a nationally representative sample. Soc Psychiatry Psychiatr Epidemiol; 2014 49: 1307-1317. [PubMed: 24081325]

10. Forbush KT \& Watson D. The structure of common and uncommon mental disorders. Psychol Med; 2013 43: 97-108. [PubMed: 22613885]

11. Krueger RF, Chentsova-Dutton YE, Markon KE, Goldberg DP, Ormel J. A cross-cultural study of the structure of comorbidity among common psychopathological syndromes in the general health care setting. J Abnorm Psychol; 2003 107: 216-227.

12. Keyes KM, Eaton NR, Krueger RF, Skodol AE, Wall MM, Grant BF et al. Thought disorder in the meta-structure of psychopathology. Psychol Med; 2013 43: 1673-1683. [PubMed: 23171498] 
13. Kotov R, Ruggero CJ, Krueger RF, Watson D, Yuan Q, Zimmerman M. New dimensions in the quantitative classification of mental illness. Arch Gen Psychiatry; 2011 68: 1003-1011. [PubMed: 21969458]

14. Markon KE. Modeling psychopathology structure: A symptom-level analysis of Axis I and II disorders. Psychol Med; 2010 40: 273-288. [PubMed: 19515267]

15. Røysamb E, Kendler KS, Tambs K, Orstavik RE, Neale MC, Aggen SH et al. The joint structure of DSM-IV Axis I and Axis II disorders. J Abnorm Psychol; 2011 120: 198-209. [PubMed: 21319931]

16. Wright AGC \& Simms LJ. A metastructural model of mental disorders and pathological personality traits. Psychol Med; 2015 45: 2309-2319. [PubMed: 25903065]

17. Harkness AR, Reynolds SM, Lilienfeld SO. A review of systems for psychology and psychiatry: Adaptive systems, Personality Psychopathology Five (PSY-5), and the DSM-5. J Pers Assess; 2014 96: 121-139. [PubMed: 23941204]

18. Kotov R, Gamez W, Schmidt F, Watson D. Linking “big” personality traits to anxiety, depressive, and substance use disorders: A meta-analysis. Psychol Bull; 2010 136: 768-821. [PubMed: 20804236]

19. Carragher N, Krueger RF, Eaton NR, Slade T. Disorders without borders: Current and future directions in the meta-structure of mental disorders. Soc Psychiatry Psychiatr Epidemiol; 2015 50: 339-350. [PubMed: 25557024]

20. Goldberg DP, Krueger RF, Andrews G, Hobbs MJ. Emotional disorders: Cluster 4 of the proposed meta-structure for DSM-V and ICD-11. Psychol Med; 2009 39: 2043-2059. [PubMed: 19796429]

21. Krueger RR, Eaton NR, Derringer J, Markon KE, Watson D, Skodol AE. Personality in DSM-5: Helping delineate personality disorder content and framing the metastructure. J Pers Assess; 2011 93: 325-331. [PubMed: 22804671]

22. Krueger RF \& South SC. Externalizing disorders: Cluster 5 of the proposed meta-structure for DSM-V and ICD-11. Psychol Med; 2009 39: 2061-2070. [PubMed: 19796431]

23. Krueger RF, Derringer J, Markon KE, Watson D, Skodol AE. Initial construction of a maladaptive personality trait model and inventory for DSM-5. Psychol Med; 2012 42: 1879-1890. [PubMed: 22153017]

24. Clark LA Temperament as a unifying basis for personality and psychopathology. J Abnorm Psychol; 2005 114: 505-521. [PubMed: 16351374]

25. Clark LA. Assessment and diagnosis of personality disorder: Perennial issues and emerging conceptualization. Ann Rev Psychol; 2007 58: 227-257. [PubMed: 16903806]

26. Krueger RF \& Eaton NR. Personality traits and the classification of mental disorders: Toward a more complete integration in DSM-5 and an empirical model of psychopathology. Personal Disord: Theory Res Treat; 2010 1: 97-118.

27. Widiger TA \& Samuel DB. Diagnostic categories or dimensions? A question for the Diagnostic and Statistical Manual of Mental Disorders-Fifth Edition. J Abnorm Psychol; 2005 114: 494 504. [PubMed: 16351373]

28. American Psychiatric Association. Diagnostic and statistical manual of mental disorders, Fifth Edition. Washington DC: Author, 2013.

29. Watson D, Stasik SM, Ro E, Clark LA. Integrating normal and pathological personality: Relating the DSM-5 trait-dimensional model to general traits of personality. Assess; 2013 20: 312-326.

30. World Health Organization. International Classification of Diseases, Version 11 for Mortality and Morbidity (ICD-11 MMS), 2018 Available at https://icd.who.int/

31. Mulder RT, Horwood J, Tyrer P, Carter J, Joyce PR. Validating the proposed ICD-11 domains. Personal Ment Health; 2016 10: 84-95. [PubMed: 27120419]

32. Reed GM. Progress in developing a classification of personality disorders for ICD-11. World Psychiatry; 2018 17: 227-228. [PubMed: 29856549]

33. Tyrer P, Reed GM, Crawford MJ. Classification, assessment, prevalence, and effect of personality disorder. Lancet; 2015 385: 717-726. [PubMed: 25706217]

34. Clark LA. Personality trait pathology, personality dysfunction, and daily functioning: What's shared and what's distinct? Keynote address presented at the XVth Congress of the International Society for the Study of Personality Disorder; Heidelberg, Germany, 2017. 
35. Clark LA \& Ro E. Three-pronged assessment and diagnosis of personality disorder and its consequences: Personality functioning, pathological traits, and psychosocial disability. Personal Disord: Theory Res Treat; 2014 5: 55-69.

36. Wright AGC, Thomas KM, Hopwood CJ, Markon KE, Pincus AL, Krueger RF. The hierarchical structure of DSM-5 pathological personality traits. J Abnorm Psychol; 2012 121: 951-957. [PubMed: 22448740]

37. Helle AC, Trull TJ, Widiger TA, Mullins-Sweatt SN Utilizing interview and self-report assessment of the five-factor model to examine convergence with the alternative model for personality disorders. Personal Disord: Theory Res Treat; 2017 8: 247-254.

38. Quilty LC, Ayearst L, Chmielewski M, Pollock BG, Bagby RM. The psychometric properties of the Personality Inventory for DSM-5 in an APA DSM-5 Field Trial sample. Assess; 2013 20: $362-$ 369.

39. John OP \& Srivastava S. The Big Five trait taxonomy: History, measurement, and theoretical perspectives. In: Pervin LA \& John OP eds. Handbook of personality, Second Edition, pp. 102138. New York: Guilford Press, 1999.

40. Lee K \& Ashton MC. Psychometric properties of the HEXACO-100. Assess; 2018 25: 543-556.

41. Suzuki T, Samuel DB, Pahlen S, Krueger RF. DSM-5 alternative personality disorder model traits as maladaptive extreme variants of the five-factor model: An item-response theory analysis. $\mathbf{J}$ Abnorm Psychol; 2015 124: 343-354. [PubMed: 25665165]

42. Goldberg LR, Johnson JA, Eber HW, Hogan R, Ashton MC, Cloninger CR. et al. The International Personality Item Pool and the future of public-domain personality measures. J Res Pers; 2006 40: 84-96.

43. Costa PT \& McCrae RR. Revised NEO Personality Inventory (NEO-PI-R) and NEO Five-Factor Inventory (NEO-FFI) professional manual Odessa FL: Psychological Assessment Resources, 1992.

44. Watson D, Stasik SM, Ellickson-Larew S, Stanton K. Explicating the psychopathological correlates of anomalous sleep experiences. Psychol Consciousness: Theory Res Pract; 2015 2: 57-78.

45. Ashton MC, Lee K, de Vries RE, Hendrickse J, Born PH. The maladaptive personality traits of the Personality Inventory for DSM-5 (PID-5) in relation to the HEXACO personality factors and schizotypy/dissociation. J Personal Disord; 2012 26: 641-659.

46. Watson D, Stanton K, Khoo S, Ellickson-Larew S, Stasik-O’Brien SM. Extraversion and psychopathology: A multilevel hierarchical review. J Res Pers; 2019 (advanced online publication)

47. Watson D, Stasik SM, Ellickson-Larew S, Stanton K. Extraversion and psychopathology: A facetlevel analysis. J Abnorm Psychol; 2015 124: 432-446. [PubMed: 25751628]

48. Chmielewski M, Bagby RM, Markon K, Ring AJ, Ryder AG. Openness to experience, intellect, schizotypal personality disorder, and psychoticism: Resolving the controversy. J Personal Disord; 2014 28: 483-499.

49. DeYoung CG, Grazioplene RG, Peterson JB. From madness to genius: The Openness/ Intellect trait domain as a paradoxical simplex. J Res Pers; 2012 46: 63-78.

50. Cohen J A power primer. Psychol Bull; 1992 112: 155-159. [PubMed: 19565683]

51. Depue RA \& Collins PF. Neurobiology of the structure of personality: Dopamine, facilitation of incentive motivation, and extraversion. Behav Brain Sci; 1999 22: 491-569. [PubMed: 11301519]

52. Smillie LD. Extraversion and reward processing. Curr Direct Psychol Sci; 2013 22: 167-172.

53. Watson D, Wiese D, Vaidya J, Tellegen A. The two general activation systems of affect: Structural findings, evolutionary considerations, and psychobiological evidence. J Pers Soc Psychol; 1999 76: 820-838.

54. Johnson SL, Edge MD, Holmes MK, Carver CS. The behavioral activation system and mania. Annu Review Clin Psychol; 2012 8: 243-267.

55. Stanton K, Gruber J, Watson D. Basic dimensions defining mania risk. Psychol Assess; 201729 : 304-319. [PubMed: 27254019]

56. Johnson SL, Leedom LJ, Muhtadie L. The dominance behavioral system and psychopathology: Evidence from self-report, observational, and biological studies. Psychol Bull; 2012 138: 692-743. [PubMed: 22506751] 
57. Naragon-Gainey K \& Watson D. Consensually-defined facets of personality as prospective predictors of change in depression symptoms. Assess; 2014 21: 387-403.

58. Lynch TR, Hempel RJ, \& Clark LA. Promoting radical openness and flexible control. In: Livesley WJ, Dimaggio G, Clarkin J. eds. Integrated modular treatment for personality disorder, pp. 325344. New York: Guilford Publications, 2015.

59. Simms LJ, Goldberg LR, Roberts JE, Watson D, Welte J, Rotterman JH. Computerized adaptive asssessment of personality disorder: Introducing the CAT-PD Project. J Pers Assess; 2011 93: 380-389. [PubMed: 22804677]

60. Wright AGC \& Simms LJ. On the structure of personality disorder traits; Conjoint analyses of the CAT-PD, PID-5, and NEO-PI-3 trait models. Personal Disord: Theory Res Treat; 2014 5: 43-54.

61. Hopwood CJ \& Donnellan MB. How should the internal structure of personality inventories be evaluated? Pers Soc Psychol Rev; 2010 14: 332-346. [PubMed: 20435808] 


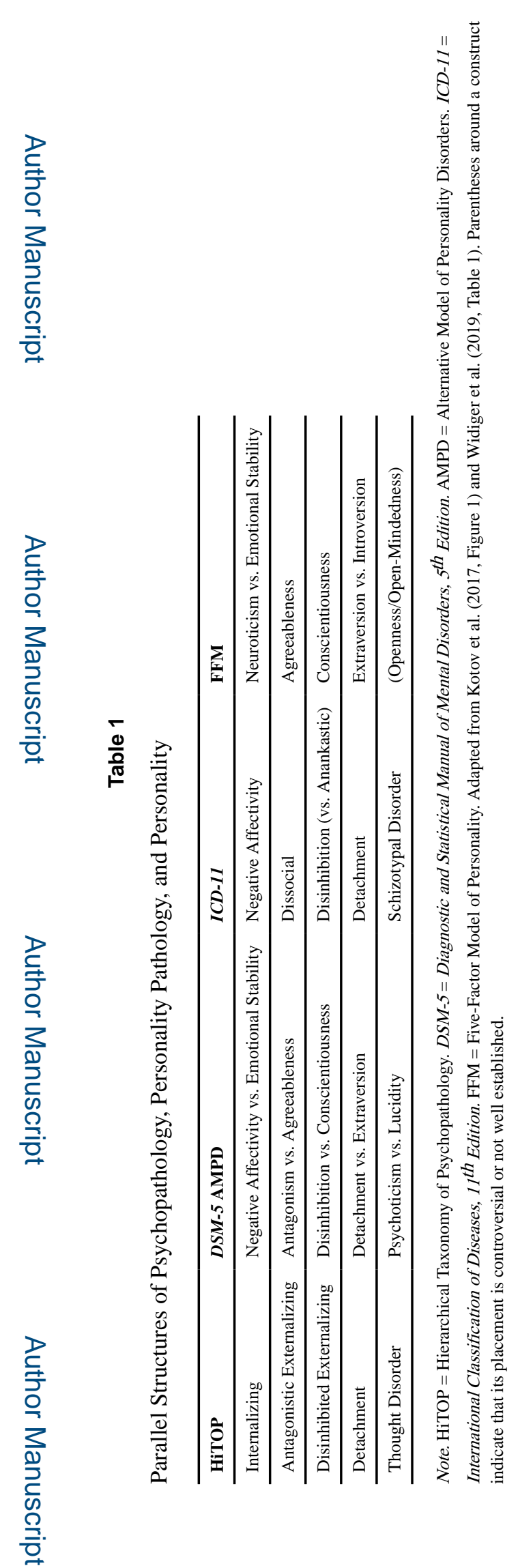

Personal Ment Health. Author manuscript; available in PMC 2021 February 01. 
Table 2

Organization of the PID-5 Facets into Five Higher Order Domains

\begin{tabular}{|c|c|c|}
\hline Preliminary Model & DSM-5 Section III Model & Triadic Model \\
\hline \multicolumn{3}{|c|}{ Negative Affectivity vs. Emotional Stability } \\
\hline \multirow{2}{*}{ Anxiousness } & Anxiousness & \multirow[t]{2}{*}{ Anxiousness } \\
\hline & Depressivity & \\
\hline Emotional Lability & Emotional Lability & \multirow[t]{4}{*}{ Emotional Lability } \\
\hline Hostility & Hostility & \\
\hline Perseveration & Perseveration & \\
\hline (Low) Restricted Affectivity & (Low) Restricted Affectivity & \\
\hline Separation Insecurity & Separation Insecurity & \multirow[t]{4}{*}{ Separation Insecurity } \\
\hline \multirow{3}{*}{ Submissiveness } & Submissiveness & \\
\hline & Suspiciousness & \\
\hline & Detachment vs. Extraversion & \\
\hline Anhedonia & Anhedonia & \multirow[t]{2}{*}{ Anhedonia } \\
\hline Depressivity & Depressivity & \\
\hline \multirow{2}{*}{ Intimacy Avoidance } & Intimacy Avoidance & \multirow[t]{2}{*}{ Intimacy Avoidance } \\
\hline & Restricted Affectivity & \\
\hline Suspiciousness & Suspiciousness & \\
\hline Withdrawal & Withdrawal & Withdrawal \\
\hline \multicolumn{3}{|c|}{ Antagonism vs. Agreeableness } \\
\hline Attention Seeking & Attention Seeking & \\
\hline Callousness & Callousness & \\
\hline Deceitfulness & Deceitfulness & Deceitfulness \\
\hline \multirow{2}{*}{ Grandiosity } & Grandiosity & \multirow[t]{2}{*}{ Grandiosity } \\
\hline & Hostility & \\
\hline Manipulativeness & Manipulativeness & Manipulativeness \\
\hline \multicolumn{3}{|c|}{ Disinhibition vs. Conscientiousness } \\
\hline Distractibility & Distractibility & Distractibility \\
\hline Impulsivity & Impulsivity & Impulsivity \\
\hline Irresponsibility & Irresponsibility & \multirow[t]{4}{*}{ Irresponsibility } \\
\hline (Low) Rigid Perfectionism & (Low) Rigid Perfectionism & \\
\hline Risk Taking & Risk Taking & \\
\hline & Psychoticism vs. Lucidity & \\
\hline Eccentricity & Eccentricity & Eccentricity \\
\hline Perceptual Dysregulation & Perceptual Dysregulation & Perceptual Dysregulation \\
\hline Unusual Beliefs & Unusual Beliefs & Unusual Beliefs \\
\hline
\end{tabular}

Note. PID-5 = Personality Inventory for DSM-5. Unusual Beliefs = Unusual Beliefs \& Experiences. 


\section{Table 3}

Correlations between FFM and PID-5 Domain Scores

\begin{tabular}{|c|c|c|c|c|c|}
\hline \multirow[b]{2}{*}{ FFM Domain Scale } & \multicolumn{5}{|c|}{ Personality Inventory for DSM-5 Domain Scale } \\
\hline & NEG & DET & ANT & DIS & PSY \\
\hline \multicolumn{6}{|l|}{ FI-FFM } \\
\hline Neuroticism & $.77^{* \dagger}$ & $.57^{\dagger}$ & .31 & .62 & $.53^{\dagger}$ \\
\hline Extraversion & -.18 & $-.53^{*}$ & .24 & -.07 & -.02 \\
\hline Agreeableness & -.31 & -.37 & $-.64^{* \dagger}$ & -.48 & -.45 \\
\hline Conscientiousness & -.38 & -.37 & -.29 & $-.70^{* \dagger}$ & -.39 \\
\hline Openness & -.05 & $-.16^{*}$ & .12 & .08 & $.17^{*}$ \\
\hline \multicolumn{6}{|l|}{$N E O-P I-3$} \\
\hline Neuroticism & $.70^{* \dagger}$ & .57 & .28 & $.64^{\dagger}$ & $.49^{\dagger}$ \\
\hline Extraversion & -.24 & $-.60^{* \dagger}$ & .11 & -.18 & -.17 \\
\hline Agreeableness & -.23 & -.26 & $-.59^{* \dagger}$ & -.37 & -.39 \\
\hline Conscientiousness & -.41 & -.42 & -.25 & $-.65^{* \dagger}$ & -.37 \\
\hline Openness & -.07 & $-.18^{*}$ & -.04 & -.04 & .03 \\
\hline \multicolumn{6}{|c|}{ Big Five Inventory (BFI) } \\
\hline Neuroticism & $.76^{* \dagger}$ & .35 & .15 & .47 & .39 \\
\hline Extraversion & -.24 & $-.67^{* \dagger}$ & .07 & -.14 & -.12 \\
\hline Agreeableness & -.35 & -.43 & $-.48 * t$ & -.45 & $-.46^{\dagger}$ \\
\hline Conscientiousness & -.39 & -.17 & -.26 & $-.70^{* \dagger}$ & -.40 \\
\hline Openness & -.13 & $-.20 *$ & -.00 & .03 & .12 \\
\hline \multicolumn{6}{|l|}{ HEXACO } \\
\hline Emotionality & $.44^{* t}$ & .13 & -.10 & .17 & .09 \\
\hline Extraversion & -.42 & $-.64^{* \dagger}$ & -.05 & -.39 & -.26 \\
\hline Honesty-Humility & -.21 & -.09 & $-.55^{* \dagger}$ & -.33 & -.25 \\
\hline Agreeableness & -.39 & -.32 & -.39 & $-.44^{*}$ & $-.35^{\dagger}$ \\
\hline Conscientiousness & -.35 & -.31 & -.30 & $-.58^{* \dagger}$ & -.30 \\
\hline Openness & $-.20^{*}$ & -.17 & -.07 & -.13 & .01 \\
\hline
\end{tabular}

Note. $N=759$ (FI-FFM), 405 (NEO-PI-3), 335 (BFI), 405 (HEXACO). Correlations with an absolute value 2.50 are in bold. FFM = Five-Factor Model. PID-5 = Personality Inventory for $D S M-5 . \mathrm{NEG}=$ Negative Affectivity. DET $=$ Detachment. ANT = Antagonism. DIS = Disinhibition. PSY $=$ Psychoticism. FI-FFM = Faceted Inventory of the Five-Factor Model. NEO-PI-3 = NEO Personality Inventory-3. BFI = Big Five Inventory. HEXACO = Revised HEXACO Personality Inventory.

Highest correlation (absolute value within \pm .01 ) in row.

${ }^{\dagger}$ Highest in column for each instrument (absolute value within \pm .01 ). 


\section{Table 4}

Promax Factor Loadings of the NEO-PI-3 and FI-FFM Domain Scales in Sample 2

\begin{tabular}{lccccc}
\hline Domain Scale & I & II & III & IV & V \\
\hline FI-FFM Conscientiousness & $\mathbf{. 9 1}$ & .03 & .01 & .02 & .02 \\
NEO-PI-3 Conscientiousness & $\mathbf{. 8 6}$ & .01 & .01 & -.10 & -.01 \\
NEO-PI-3 Agreeableness & -.04 & $\mathbf{. 8 7}$ & -.07 & -.03 & .05 \\
FI-FFM Agreeableness & .10 & $\mathbf{. 8 4}$ & .07 & -.01 & -.06 \\
FI-FFM Extraversion & .01 & -.08 & $\mathbf{. 8 8}$ & .02 & .01 \\
NEO-PI-3 Extraversion & .00 & .08 & $\mathbf{. 8 4}$ & -.05 & .05 \\
FI-FFM Neuroticism & .00 & -.07 & .00 & $\mathbf{. 8 5}$ & -.01 \\
NEO-PI-3 Neuroticism & -.12 & .00 & -.04 & $\mathbf{. 8 2}$ & .02 \\
NEO-PI-3 Openness & .01 & .06 & -.02 & .03 & $\mathbf{. 8 3}$ \\
FI-FFM Openness & .00 & -.06 & .07 & -.03 & $\mathbf{. 8 1}$ \\
\hline
\end{tabular}

Note. $N=433$. Loadings $\geq .40$ are in bold. NEO-PI-3 = NEO Personality Inventory-3.

FI-FFM = Faceted Inventory of the Five-Factor Model. 
Table 5

Correlations between FFM Domain Factor Scores and the PID-5 Facet Scales

\begin{tabular}{|c|c|c|c|c|c|}
\hline PID-5 Facet Scale & NEUR & EXTRA & AGREE & CON & OPEN \\
\hline \multicolumn{6}{|l|}{ Negative Affectivity Facets } \\
\hline Anxiousness & $.69^{* \dagger}$ & -.29 & -.27 & -.38 & -.06 \\
\hline Emotional Lability & $.63^{*}$ & -.19 & -.31 & -.37 & -.05 \\
\hline Separation Insecurity & $.50 *$ & -.14 & -.29 & -.35 & -.05 \\
\hline \multicolumn{6}{|l|}{ Detachment Facets } \\
\hline Anhedonia & $.66^{*}$ & $-.59{ }^{\dagger}$ & -.31 & -.53 & -.21 \\
\hline Withdrawal & $.52 *$ & $-.52 *$ & -.30 & -.33 & -.23 \\
\hline Intimacy Avoidance & $.35^{*}$ & -.28 & -.26 & -.28 & -.09 \\
\hline \multicolumn{6}{|l|}{ Antagonism Facets } \\
\hline Deceitfulness & .39 & .06 & $-.64^{*}$ & -.39 & .03 \\
\hline Manipulativeness & .23 & .22 & $-.54^{*}$ & -.23 & .12 \\
\hline Grandiosity & .12 & .22 & $-.39^{*}$ & .02 & .05 \\
\hline \multicolumn{6}{|l|}{ Disinhibition Facets } \\
\hline Distractibility & $.64^{*}$ & -.27 & -.35 & $-.63^{* \dagger}$ & -.01 \\
\hline Irresponsibility & .53 & -.15 & -.52 & $-.56^{*}$ & -.03 \\
\hline Impulsivity & .48 & .02 & -.43 & $-.50^{*}$ & .11 \\
\hline \multicolumn{6}{|l|}{ Psychoticism Facets } \\
\hline Perceptual Dysregulation & $.53^{*}$ & -.18 & -.40 & -.35 & -.00 \\
\hline Unusual Beliefs \& Experiences & $.37^{*}$ & -.06 & -.35 & -.27 & .04 \\
\hline Eccentricity & $.48^{*}$ & -.06 & -.44 & -.37 & .10 \\
\hline \multicolumn{6}{|l|}{ Other Scales } \\
\hline Depressivity & $.68^{* \dagger}$ & -.41 & -.33 & -.52 & -.05 \\
\hline Hostility & $.61 *$ & -.12 & -.59 & -.39 & -.03 \\
\hline Perseveration & $.54^{*}$ & -.18 & -.35 & -.39 & -.01 \\
\hline Rigid Perfectionism & $.31^{*}$ & -.06 & -.16 & .00 & -.08 \\
\hline Submissiveness & $.25^{*}$ & -.15 & .01 & -.21 & .01 \\
\hline Attention Seeking & .15 & $.42^{*}$ & $-.43^{*}$ & -.12 & .30 \\
\hline Risk Taking & .08 & $.43^{*}$ & -.37 & -.17 & $.41^{\dagger}$ \\
\hline Callousness & .42 & .04 & $-.66^{* t}$ & -.32 & .06 \\
\hline Suspiciousness & .51 & -.17 & $-.53^{*}$ & -.32 & -.13 \\
\hline Restricted Affectivity & .24 & -.20 & $-.34^{*}$ & -.21 & -.03 \\
\hline
\end{tabular}

$N=405$. Correlations with an absolute value 2.40 are in bold. FFM $=$ Five-Factor Model. PID- $5=$ Personality Inventory for DSM-5. NEUR $=$ Neuroticism. EXTRA $=$ Extraversion. AGREE $=$ Agreeableness. CON $=$ Conscientiousness. OPEN $=$ Openness.

Highest correlation (absolute value within \pm .01 ) in row.

Personal Ment Health. Author manuscript; available in PMC 2021 February 01. 
${ }^{\dagger}$ Highest correlation (absolute value within \pm .01 ) in column. 
Table 6

Correlations between Neuroticism Facets and Selected PID-5 Scales

\begin{tabular}{|c|c|c|c|c|c|c|}
\hline \multirow[b]{2}{*}{ Facet Scale } & \multicolumn{6}{|c|}{ Personality Inventory for DSM-5 Scales } \\
\hline & NEG & Anxious $^{a}$ & Depress & Hostility & Lability $^{a}$ & Separ $^{a}$ \\
\hline \multicolumn{7}{|l|}{ FI-FFM } \\
\hline Anxiety & $.74^{* \dagger}$ & $.75^{* \dagger}$ & .57 & .46 & $.65^{\dagger}$ & .45 \\
\hline Depression & $.74^{\dagger}$ & .72 & $.78^{* \dagger}$ & .59 & $.65^{\dagger}$ & $.51^{\dagger}$ \\
\hline Anger Proneness & .64 & .58 & .58 & $.76^{* t}$ & $.65^{\dagger}$ & .39 \\
\hline Somatic Complaints & $.58^{*}$ & $.57^{*}$ & .54 & .45 & .52 & .38 \\
\hline Envy & $.54 *$ & .51 & .48 & .51 & .44 & .42 \\
\hline \multicolumn{7}{|l|}{ NEO-PI-3 } \\
\hline Anxiety & $.65^{\dagger}$ & $.68^{* \dagger}$ & .50 & .43 & $.55^{\dagger}$ & .41 \\
\hline Depression & .63 & .61 & $.69^{* \dagger}$ & .45 & $.55^{\dagger}$ & $.47^{\dagger}$ \\
\hline Angry Hostility & .52 & .47 & .42 & $.65^{* \dagger}$ & .53 & .35 \\
\hline Vulnerability & .53 & .53 & $.55^{*}$ & .38 & .45 & .39 \\
\hline Self-Consciousness & .51 & .51 & $.59^{*}$ & .30 & .40 & .40 \\
\hline Impulsiveness & $.47^{*}$ & .44 & .40 & .45 & .42 & .34 \\
\hline \multicolumn{7}{|l|}{$I P I P-N E O$} \\
\hline Anxiety &.- & $.79^{* t}$ & .57 & .48 & .60 & $.48^{\dagger}$ \\
\hline Depression & .- & .66 & $.80^{* \dagger}$ & .48 & .60 & $.47^{\dagger}$ \\
\hline Anger & .- & .54 & .44 & $.70^{* \dagger}$ & .56 & .37 \\
\hline Vulnerability & .- & $.68^{*}$ & .57 & .45 & $.62^{\dagger}$ & $.48^{\dagger}$ \\
\hline Self-Consciousness & .- & $.51^{*}$ & .49 & .32 & .39 & .35 \\
\hline Immoderation &.- & .44 & .38 & .41 & $.46^{*}$ & .36 \\
\hline \multicolumn{7}{|l|}{ HEXACO } \\
\hline Anxiety & $.48^{\dagger}$ & $.52^{* \dagger}$ & $.35^{\dagger}$ & $.30 t$ & $.41^{\dagger}$ & $.29^{\dagger}$ \\
\hline Dependence & $.28^{*}$ & .25 & .11 & .11 & .24 & .24 \\
\hline Sentimentality & .21 & .16 & .02 & .01 & $.26^{*}$ & .12 \\
\hline Fearfulness & $.18^{*}$ & $.19^{*}$ & .07 & -.03 & .17 & .09 \\
\hline
\end{tabular}

Note. $N=759$ (FI-FFM), 405 (NEO-PI-3), 3,517 (IPIP-NEO), 405 (HEXACO). Correlations with an absolute value 2.50 are in bold. PID-5 = Personality Inventory for DSM-5. NEG = Negative Affectivity (domain scale). Anxious = Anxiousness. Depress = Depressivity. Lability = Emotional Lability. Separ $=$ Separation Insecurity. FI-FFM = Faceted Inventory of the Five-Factor Model. NEO-PI-3 = NEO Personality Inventory-3. IPIP-NEO = International Personality Item Pool-NEO PI-R. HEXACO = HEXACO Personality Inventory-Revised.

${ }^{a}$ Current marker of the PID-5 Negative Affectivity domain.

Highest correlation in row (absolute value within \pm .01 ).

${ }^{\dagger}$ Highest in column for each instrument (absolute value within \pm .01 ). 
Table 7

Correlations between Extraversion Facets and Selected PID-5 Scales

\begin{tabular}{|c|c|c|c|c|c|c|}
\hline \multirow[b]{2}{*}{ Facet Scale } & \multicolumn{6}{|c|}{ Personality Inventory for DSM-5 Scales } \\
\hline & DET & With $^{a}$ & Anhed $^{a}$ & $\operatorname{Intim}^{a}$ & Risk & Atten \\
\hline \multicolumn{7}{|l|}{$F I-F F M$} \\
\hline Sociability & $-.63^{\dagger}$ & $-.68^{* \dagger}$ & -.47 & $-.39^{\dagger}$ & .09 & .19 \\
\hline Positive Temperament & -.56 & -.43 & $-.67^{* \dagger}$ & -.29 & .23 & .23 \\
\hline Venturesomeness &.- .33 &.- .33 & -.29 & -.17 & $.56^{* \dagger}$ & .45 \\
\hline Ascendance & -.28 & -.30 & -.25 & -.13 & .34 & $.56 * \dagger$ \\
\hline Frankness & -.15 & -.17 & -.14 & -.02 & $.31^{*}$ & $.32 *$ \\
\hline \multicolumn{7}{|l|}{ NEO-PI-3 } \\
\hline Gregariousness & -.50 & $-.54^{* t}$ & -.41 & $-.27^{\dagger}$ & .19 & .26 \\
\hline Warmth & $-.53^{* \dagger}$ & $-.53^{* \dagger}$ & -.51 & $-.26^{\dagger}$ & .11 & .14 \\
\hline Positive Emotions & -.51 & -.42 & $-.62^{* \dagger}$ & -.24 & .14 & .16 \\
\hline Activity & -.35 & -.30 & $-.44^{*}$ & -.14 & .32 & .26 \\
\hline Excitement-Seeking & -.24 & -.18 & -.23 & -.22 & $.56^{* \dagger}$ & $.36^{\dagger}$ \\
\hline Assertiveness & $-.35^{*}$ & $-.35^{*}$ & $-.35^{*}$ & -.16 & .24 & .31 \\
\hline \multicolumn{7}{|l|}{$I P I P-N E O$} \\
\hline Friendliness & - - & $-.79^{* \dagger}$ & $-.63^{\dagger}$ & $-.37^{\dagger}$ & .07 & .07 \\
\hline Gregariousness & - - & $-.70^{*}$ & -.48 & $-.37{ }^{\dagger}$ & .29 & .28 \\
\hline Cheerfulness & .- & -.48 & $-.62^{* \dagger}$ & -.28 & .25 & .22 \\
\hline Activity Level & - & -.26 & $-.36^{*}$ & -.15 & .14 & .06 \\
\hline Excitement-Seeking & .- & -.18 & -.16 & -.14 & $.73^{* \dagger}$ & $.52^{\dagger}$ \\
\hline Assertiveness & .- & -.39 & -.35 & -.20 & .36 & $.49 *$ \\
\hline \multicolumn{7}{|l|}{ HEXACO } \\
\hline Sociability & -.53 & $-.56^{* \dagger}$ & -.44 & -.28 & .16 & .28 \\
\hline Social Self-Esteem & -.53 & -.39 & $-.65^{*}$ & -.29 & -.00 & -.01 \\
\hline Liveliness & $-.58^{\dagger}$ & -.45 & $-.69^{* \dagger}$ & $-.31^{\dagger}$ & .08 & .05 \\
\hline Social Boldness & -.34 & $-.39 *$ & -.32 & -.10 & $.25^{\dagger}$ & $.33^{\dagger}$ \\
\hline
\end{tabular}

Note. $N=759$ (FI-FFM), 405 (NEO-PI-3), 3,517 (IPIP-NEO), 405 (HEXACO). Correlations with an absolute value 2.30 are in bold. PID-5 = Personality Inventory for DSM-5. DET = Detachment (domain scale). With = Withdrawal. Anhed = Anhedonia. Intim = Intimacy Avoidance. Risk $=$ Risk Taking. Atten $=$ Attention Seeking. FI-FFM $=$ Faceted Inventory of the Five-Factor Model. NEO-PI-3 = NEO Personality Inventory-3. IPIP$\mathrm{NEO}=$ International Personality Item Pool-NEO PI-R. HEXACO = HEXACO Personality Inventory-Revised.

${ }^{a}$ Current marker of the PID-5 Detachment domain.

Highest correlation in row (absolute value within \pm .01 ).

${ }^{\dagger}$ Highest in column for each instrument (absolute value within \pm .01 ).

Personal Ment Health. Author manuscript; available in PMC 2021 February 01. 
Table 8

Correlations between Agreeableness \& Honesty-Humility Facets and Selected PID-5 Scales

\begin{tabular}{|c|c|c|c|c|c|c|c|c|}
\hline \multirow[b]{2}{*}{ Facet Scale } & \multicolumn{8}{|c|}{ Personality Inventory for $D S M-5$ Scales } \\
\hline & ANT & Deceit $^{a}$ & Manip $^{a}$ & Callous & Host & Suspic & Grand $^{a}$ & Atten \\
\hline \multicolumn{9}{|l|}{ FI-FFM } \\
\hline Straightforwardness & $-.69^{\dagger}$ & $-.71^{* \dagger}$ & $-.64^{\dagger}$ & $-.62^{\dagger}$ & $-.56^{\dagger}$ & -.36 & -.37 & -.45 \\
\hline Empathy & -.41 & -.41 & -.33 & $-.53 *$ & -.36 & -.24 & -.29 & -.19 \\
\hline Trust & -.32 & -.32 & -.23 & -.43 & -.50 & $-.65^{* \dagger}$ & -.24 & -.16 \\
\hline Modesty & $-.52^{*}$ & -.48 & -.45 & -.48 & -.39 & -.30 & $-.42^{\dagger}$ & $-.51 * \dagger$ \\
\hline \multicolumn{9}{|l|}{$N E O-P I-3$} \\
\hline Straightforwardness & $-.62^{\dagger}$ & $-.65^{* \dagger}$ & $-.60 \dagger$ & $-.55 \dagger$ & -.39 & -.28 & -.28 & -.43 \\
\hline Compliance & -.39 & -.35 & -.36 & -.50 & $-.58^{* t}$ & -.38 & -.28 & -.30 \\
\hline Trust & -.26 & -.29 & -.19 & -.36 & -.45 & $-.62 * t$ & -.15 & -.03 \\
\hline Modesty & -.41 & -.27 & -.33 & -.24 & -.09 & -.14 & $-.48^{* \dagger}$ & $-.45^{\dagger}$ \\
\hline Altruism & -.37 & $-.45^{*}$ & -.29 & $-.46^{*}$ & -.32 & -.24 & -.14 & -.17 \\
\hline Tender mindedness & -.16 & -.16 & -.11 & $-.21^{*}$ & -.07 & -.06 & -.14 & -.03 \\
\hline \multicolumn{9}{|l|}{$I P I P-N E O$} \\
\hline Morality & - & $-.78^{* \dagger}$ & $-.74^{\dagger}$ & -.62 & -.55 & -.46 & -.55 & -.57 \\
\hline Cooperation & - & -.58 & -.54 & -.59 & $-.66^{* \dagger}$ & -.44 & -.44 & -.47 \\
\hline Trust & - & -.43 & -.30 & -.45 & -.52 & $-.66^{* \dagger}$ & -.28 & -.23 \\
\hline Modesty & - & -.42 & -.55 & -.32 & -.26 & -.17 & $-.62^{\dagger}$ & $-.68^{* \dagger}$ \\
\hline Altruism & - & -.48 & -.35 & $-.65^{* \dagger}$ & -.53 & -.43 & -.38 & -.24 \\
\hline Sympathy & - & -.45 & -.41 & $-.59^{*}$ & -.44 & -.33 & -.44 & -.30 \\
\hline \multicolumn{9}{|l|}{ HEXACO } \\
\hline Fairness (H) & $-.50^{\dagger}$ & $-.54^{* \dagger}$ & $-.43^{\dagger}$ & $-.51^{\dagger}$ & -.36 & -.32 & -.24 & -.34 \\
\hline Sincerity $(\mathrm{H})$ & -.41 & $-.45^{*}$ & -.33 & -.32 & -.26 & -.19 & -.22 & -.29 \\
\hline Modesty (H) & -.38 & -.30 & -.26 & -.30 & -.21 & -.22 & $-.43^{* \dagger}$ & $-.36^{\dagger}$ \\
\hline Flexibility (A) & -.33 & -.34 & -.33 & -.38 & $-.48^{*}$ & -.28 & -.14 & -.27 \\
\hline Patience (A) & -.31 & -.32 & -.29 & -.42 & $-.63^{* \dagger}$ & $-.38^{\dagger}$ & -.17 & -.21 \\
\hline Gentleness (A) & -.28 & -.28 & -.25 & $-.33^{*}$ & $-.32^{*}$ & -.21 & -.16 & -.17 \\
\hline Forgivingness (A) & -.27 & -.31 & -.24 & -.33 & $-.46^{*}$ & -.35 & -.10 & -.19 \\
\hline Greed Avoidance (H) & -.22 & -.18 & -.17 & -.20 & -.12 & -.19 & -.21 & $-.24^{*}$ \\
\hline
\end{tabular}

Note. $N=759$ (FI-FFM), 405 (NEO-PI-3), 3,517 (IPIP-NEO), 405 (HEXACO). Correlations with an absolute value $\geq .50$ are in bold. PID-5= Personality Inventory for DSM-5. ANT = Antagonism (domain scale). Deceit $=$ Deceitfulness. Manip $=$ Manipulativeness. Callous $=$ Callousness. Host $=$ Hostility. Suspic $=$ Suspiciousness. Grand $=$ Grandiosity. Atten $=$ Attention Seeking. FI-FFM $=$ Faceted Inventory of the Five-Factor Model. NEO-PI-3 = NEO Personality Inventory-3. IPIP-NEO = International Personality Item Pool-NEO PI-R. HEXACO = HEXACO Personality Inventory-Revised. $\mathrm{H}=$ Honesty-Humility facet. $\mathrm{A}=$ Agreeableness facet.

${ }^{a}$ Current marker of the PID-5 Antagonism domain.

Personal Ment Health. Author manuscript; available in PMC 2021 February 01. 
Highest correlation in row (absolute value within \pm .01 ).

${ }^{\dagger}$ Highest in column for each instrument (absolute value within \pm .01 ). 


\section{Table 9}

Correlations between Conscientiousness Facets and Selected PID-5 Scales

\begin{tabular}{|c|c|c|c|c|}
\hline \multirow[b]{2}{*}{ Facet Scale } & \multicolumn{4}{|c|}{ Personality Inventory for DSM-5 Scales } \\
\hline & DIS & Irrespon $^{a}$ & Impulsive $^{a}$ & Distract $^{a}$ \\
\hline \multicolumn{5}{|l|}{ FI-FFM } \\
\hline Dutifulness & -.59 & $-.66^{* \dagger}$ & -.42 & -.48 \\
\hline Deliberation & $-.64^{\dagger}$ & -.57 & $-.70^{* \dagger}$ & -.45 \\
\hline Self-Discipline & $-.63^{\dagger}$ & -.49 & -.42 & $-.65^{* \dagger}$ \\
\hline Order & $-.49^{*}$ & -.40 & -.36 & -.47 \\
\hline Achievement Striving & -.33 & -.23 & -.19 & $-.37^{*}$ \\
\hline \multicolumn{5}{|l|}{$N E O-P I-3$} \\
\hline Dutifulness & -.50 & $-.53^{* \dagger}$ & -.37 & -.42 \\
\hline Deliberation & -.59 & -.46 & $-.65^{* \dagger}$ & -.44 \\
\hline Self-Discipline & $-.62^{* \dagger}$ & $-.52^{\dagger}$ & -.40 & $-.62^{* \dagger}$ \\
\hline Competence & $-.55^{*}$ & -.45 & -.42 & -.52 \\
\hline Order & $-.43^{*}$ & -.37 & -.30 & -.41 \\
\hline Achievement Striving & -.37 & -.33 & -.19 & $-.40^{*}$ \\
\hline \multicolumn{5}{|l|}{$I P I P-N E O$} \\
\hline Dutifulness &.- & $-.71^{* \dagger}$ & -.55 & -.55 \\
\hline Cautiousness & - - & -.52 & $-.76^{* \dagger}$ & -.55 \\
\hline Self-Discipline &.- & -.54 & -.42 & $-.68^{* \dagger}$ \\
\hline Self-Efficacy & - - & -.46 & -.39 & $-.52^{*}$ \\
\hline Orderliness & - - & $-.43^{*}$ & -.36 & -.39 \\
\hline Achievement-Striving &.- & -.41 & -.30 & $-.43^{*}$ \\
\hline \multicolumn{5}{|l|}{ HEXACO } \\
\hline Prudence & $-.64^{* t}$ & $-.56^{\dagger}$ & $-.61^{\dagger}$ & $-.51^{\dagger}$ \\
\hline Diligence & $-.41^{*}$ & -.38 & -.22 & $-.42^{*}$ \\
\hline Organization & $-.44^{*}$ & -.36 & -.31 & $-.43^{*}$ \\
\hline Perfectionism & -.23 & $-.29^{*}$ & -.17 & -.17 \\
\hline
\end{tabular}

Note. $N=759$ (FI-FFM), 405 (NEO-PI-3), 3,517 (IPIP-NEO) 405 (HEXACO). Correlations with an absolute value $\geq .50$ are in bold. PID-5 = Personality Inventory for DSM-5. DIS = Disinhibition (domain scale). Irrespon = Irresponsibility. Impulsive = Impulsivity. Distract =

Distractibility. FI-FFM = Faceted Inventory of the Five-Factor Model. NEO-PI-3 = NEO Personality Inventory-3. IPIP-NEO = International Personality Item Pool-NEO PI-R. HEXACO = HEXACO Personality Inventory-Revised.

${ }^{a}$ Current marker of the PID-5 Disinhibition domain.

Highest correlation in row (absolute value within \pm .01 ).

${ }^{\dagger}$ Highest in column for each instrument (absolute value within \pm .01 ). 
Table 10

Correlations between Openness Facets and Selected PID-5 Scales

\begin{tabular}{|c|c|c|c|c|}
\hline \multirow[b]{2}{*}{ Facet Scale } & \multicolumn{4}{|c|}{ Personality Inventory for DSM-5 Scales } \\
\hline & PSY & Perceptual $^{a}$ & Unusual $^{a}$ & Eccentric $^{a}$ \\
\hline \multicolumn{5}{|l|}{$F I-F F M$} \\
\hline Nontraditionalism & $.24^{\dagger}$ & $.15^{\dagger}$ & $.17^{\dagger}$ & $.28^{* \dagger}$ \\
\hline Novel Experience Seeking & .10 & .04 & .08 & $.12^{*}$ \\
\hline Intellectance & .04 & -.00 & $.10^{*}$ & .03 \\
\hline \multicolumn{5}{|l|}{ NEO-PI-3 } \\
\hline Fantasy & $.20 * \dagger$ & .15 & .17 & $.21^{* \dagger}$ \\
\hline Feelings & $.09^{*}$ & .05 & .08 & $.10^{*}$ \\
\hline Aesthetics & .05 & .06 & $.09^{*}$ & .01 \\
\hline Ideas & -.02 & $-.05^{*}$ & -.02 & .01 \\
\hline Values & -.10 & -.13 & $-.15^{* \dagger}$ & -.05 \\
\hline Actions & -.15 & $-.17^{* \dagger}$ & $-.14^{\dagger}$ & -.11 \\
\hline \multicolumn{5}{|l|}{ IPIP-NEO } \\
\hline Imagination & - - & $.34{ }^{\dagger}$ & $.33^{\dagger}$ & $.40 * \dagger$ \\
\hline Liberalism & - - & .17 & .11 & $.21^{*}$ \\
\hline Emotionality & .- & .05 & $.07^{*}$ & .04 \\
\hline Intellect & - & -.06 & $.08^{*}$ & .04 \\
\hline Adventurousness & - - & $-.08^{*}$ & .01 & -.02 \\
\hline Artistic Interests & .- & $-.11^{*}$ & .02 & -.09 \\
\hline \multicolumn{5}{|l|}{ HEXACO } \\
\hline Unconventionality & $.12^{* \dagger}$ & $.09^{\dagger}$ & $.10^{\dagger}$ & $.11^{* \dagger}$ \\
\hline Creativity & .06 & .02 & $.10^{* \dagger}$ & .06 \\
\hline Aesthetic Appreciation & -.05 & -.05 & .00 & $-.07 *$ \\
\hline Inquisitiveness & $-.06^{*}$ & $-.06^{*}$ & -.02 & $-.06^{*}$ \\
\hline
\end{tabular}

Note. $N=759$ (FI-FFM), 405 (NEO-PI-3), 3,517 (IPIP-NEO) 405 (HEXACO). Correlations with an absolute value $\geq .30$ are in bold. PID-5= Personality Inventory for DSM-5. PSY $=$ Psychoticism $($ domain scale). Perceptual $=$ Perceptual Dysregulation. Unusual $=$ Unusual Beliefs $\&$ Experiences. Eccentric $=$ Eccentricity. FI-FFM $=$ Faceted Inventory of the Five-Factor Model. NEO-PI-3 = NEO Personality Inventory-3. IPIP$\mathrm{NEO}=$ International Personality Item Pool-NEO PI-R. HEXACO = HEXACO Personality Inventory-Revised.

${ }^{a}$ Current marker of the PID-5 Psychoticism domain.

* Highest correlation in row (absolute value within \pm .01 ).

${ }^{\dagger}$ Highest in column for each instrument (absolute value within \pm .01 ). 
Table 11

Personality and Revised PID-5 Domain Correlations

\begin{tabular}{|c|c|c|c|c|c|c|}
\hline \multirow[b]{2}{*}{ FFM Scale } & \multicolumn{2}{|c|}{$\underline{\text { Negative Affect }}$} & \multicolumn{2}{|c|}{ Detachment } & \multicolumn{2}{|c|}{ Antagonism } \\
\hline & Curr & $\operatorname{Rev}$ & Curr & Rev & Curr & Rev \\
\hline \multicolumn{7}{|l|}{$F I-F F M$} \\
\hline Neuroticism & $.77^{\dagger}$ & $.83^{* \dagger}$ & $.57^{\dagger}$ & .29 & .31 & .44 \\
\hline Extraversion & -.18 & -.24 & -.53 & $-.76^{* \dagger}$ & .24 & .14 \\
\hline Agreeableness & -.31 & -.43 & -.37 & .07 & $-.64^{\dagger}$ & $-.73^{* \dagger}$ \\
\hline Conscientiousness & -.38 & $-.46^{*}$ & -.37 & -.06 & -.29 & -.36 \\
\hline Openness & -.05 & -.02 & -.16 & $-.38^{*}$ & .12 & .08 \\
\hline \multicolumn{7}{|l|}{$N E O-P I-3$} \\
\hline Neuroticism & $.70^{\dagger}$ & $.74^{* \dagger}$ & .57 & .33 & .28 & .40 \\
\hline Extraversion & -.24 & -.36 & $-.60^{\dagger}$ & $-.74^{* \dagger}$ & .11 & -.01 \\
\hline Agreeableness & -.23 & -.35 & -.26 & .12 & $-.599^{\dagger}$ & $-.68^{* \dagger}$ \\
\hline Conscientiousness & -.41 & $-.50 *$ & -.42 & -.18 & -.25 & -.33 \\
\hline Openness & -.07 & -.07 & -.18 & $-.33^{*}$ & -.04 & -.07 \\
\hline \multicolumn{7}{|c|}{ Big Five Inventory (BFI) } \\
\hline Neuroticism & $.76^{\dagger}$ & $.78^{* \dagger}$ & .35 & .35 & .15 & .27 \\
\hline Extraversion & -.24 & -.29 & $-.67^{* t}$ & $-.61^{\dagger}$ & .07 & -.02 \\
\hline Agreeableness & -.35 & -.49 & -.43 & -.06 & $-.48^{\dagger}$ & $-.61^{* \dagger}$ \\
\hline Conscientiousness & -.39 & $-.43^{*}$ & -.17 & .03 & -.26 & -.32 \\
\hline Openness & -.13 & -.09 & -.20 & $-.30^{*}$ & -.00 & -.06 \\
\hline \multicolumn{7}{|l|}{ HEXACO } \\
\hline Emotionality & $.44^{* \dagger}$ & .30 & .13 & .27 & -.10 & -.07 \\
\hline Extraversion & -.42 & $-.53^{\dagger}$ & $-.64^{* \dagger}$ & $-.62^{\dagger}$ & -.05 & -.18 \\
\hline Honesty-Humility & -.21 & -.24 & -.09 & .24 & $-.55^{\dagger}$ & $-.57^{* \dagger}$ \\
\hline Agreeableness & -.39 & $-.49^{*}$ & -.32 & -.03 & -.39 & $-.49^{*}$ \\
\hline Conscientiousness & -.35 & $-.41^{*}$ & -.31 & -.08 & -.30 & -.36 \\
\hline Openness & -.20 & -.16 & -.17 & $-.27^{*}$ & -.07 & -.08 \\
\hline
\end{tabular}

Note. $N=759$ (FI-FFM), 405 (NEO-PI-3), 335 (BFI), 405 (HEXACO). Correlations with an absolute value 2.50 are in bold. PID-5 = Personality Inventory for DSM-5. Curr = Current PID-5 domain (Triadic model). Rev = Revised. FI-FFM = Faceted Inventory of the Five-Factor Model. NEOPI-3 = NEO Personality Inventory-3. BFI = Big Five Inventory. HEXACO = Revised HEXACO Personality Inventory.

*Highest correlation in row (absolute value within \pm .01 ).

${ }^{\dagger}$ Highest in column for each instrument (absolute value within \pm .01 ). 


\section{Table 12}

Promax Loadings of the PID-5 and FI-FFM Facet Scales

\begin{tabular}{|c|c|c|c|c|}
\hline & $\mathbf{I}$ & II & III & IV \\
\hline PID-5 Anxiousness & .94 & -.08 & .03 & .11 \\
\hline FI-FFM Anxiety & .94 & -.29 & .03 & -.03 \\
\hline PID-5 Emotional Lability & .86 & -.06 & .16 & .01 \\
\hline FI-FFM Depression & .79 & .01 & -.15 & -.13 \\
\hline FI-FFM Somatic Complaints & .73 & -.14 & -.04 & -.06 \\
\hline PID-5 Depressivity & .73 & .08 & -.17 & -.05 \\
\hline FI-FFM Anger Proneness & .67 & .20 & -.02 & -.07 \\
\hline PID-5 Anhedonia & .54 & .16 & -.41 & -.08 \\
\hline FI-FFM Envy & .52 & .18 & .06 & -.09 \\
\hline PID-5 Suspiciousness & .52 & .41 & -.05 & .14 \\
\hline PID-5 Distractibility & .51 & .01 & .03 & -.40 \\
\hline PID-5 Callousness & .02 & .82 & -.00 & .00 \\
\hline PID-5 Deceitfulness & .10 & .68 & .16 & -.10 \\
\hline PID-5 Manipulativeness & .01 & .67 & .30 & -.01 \\
\hline PID-5 Grandiosity & .06 & .64 & .22 & .26 \\
\hline PID-5 Hostility & .52 & .50 & .04 & .04 \\
\hline FI-FFM Trust & -.25 & -.49 & .17 & -.08 \\
\hline FI-FFM Modesty & .09 & -.60 & -.18 & .12 \\
\hline FI-FFM Straightforwardness & -.02 & -.66 & -.13 & .21 \\
\hline FI-FFM Empathy & .39 & -.76 & .29 & .23 \\
\hline FI-FFM Venturesomeness & -.06 & .18 & .72 & -.02 \\
\hline FI-FFM Sociability & -.06 & -.38 & .67 & -.11 \\
\hline FI-FFM Ascendance & -.06 & .29 & .65 & .15 \\
\hline FI-FFM Positive Temperament & -.23 & .02 & .63 & .27 \\
\hline PID-5 Attention Seeking & .19 & .37 & .58 & -.03 \\
\hline FI-FFM Frankness & .05 & .20 & .48 & .08 \\
\hline PID-5 Risk Taking & -.06 & .38 & .45 & -.23 \\
\hline PID-5 Withdrawal & .31 & .43 & -.53 & .15 \\
\hline FI-FFM Self-Discipline & -.15 & .04 & .03 & .76 \\
\hline FI-FFM Order & .04 & .01 & .07 & .73 \\
\hline FI-FFM Dutifulness & -.01 & -.18 & .11 & .67 \\
\hline FI-FFM Deliberation & -.04 & -.22 & -.22 & .61 \\
\hline FI-FFM Achievement Striving & -.00 & .13 & .40 & .60 \\
\hline PID-5 Impulsivity & .33 & .24 & .29 & -.36 \\
\hline PID-5 Irresponsibility & .23 & .37 & .07 & -.40 \\
\hline
\end{tabular}

Note. $N=759$. Loadings with an absolute value 2.35 are in bold. PID-5 = Personality Inventory for DSM-5. FI-FFM = Faceted Inventory of the Five-Factor Model. 